\title{
Analysis of Magnetic Resonance Images of Disk Positions and Deformities in 1,265 Patients with Temporomandibular Disorder
}

\author{
Yong-Suk Choi ${ }^{1,2}$, Jun-ichi Asaumi ${ }^{1, *}$, Miki Hisatomi ${ }^{1}$, Teruhisa Unetsubo ${ }^{1}$, Yoshinobu Yanagi ${ }^{1}$, \\ Hidenobu Matsuzaki ${ }^{1}$, Hironobu Konouchi ${ }^{1}$, Eui-Hwan Hwang ${ }^{2}$ and Sang-Rae Lee ${ }^{2}$
}

${ }^{I}$ Department of Oral and Maxillofacial Radiology, Field of Tumor Biology, Okayama University Graduate School of Medicine, Dentistry and Pharmaceutical Sciences, Okayama, Japan and ${ }^{2}$ Deprartment of Oral and Maxillofacial Radiology, College of Dentistry, Kyung Hee University, Seoul, Korea

\begin{abstract}
Objectives: To compare MRI manifestations according to gender and age and to identify correlations between clinical manifestations and MRI findings in patients with temporomandibular disorder (TMD) as based on a large series.

Materials and Methods: Fat suppressed oblique sagittal images of the open and closed mouth were acquired, and MRI scanning parameters were applied.

Results: The patients consisted of 946 females (average, 36.6 years old), and 319 males (average, 34.3 years old). In all TMD patients, 945 had symptoms in the unilateral temporomandibular joint (TMJ) and 320 in the bilateral TMJ. There were significant differences in the distribution of disk positions based on age, regardless of gender, in the unilaterally and bilaterally symptomatic groups; however, the results were not significant in the asymptomatic group. There were significant differences with respect to the distribution of disk positions between males and females in asymptomatic group and in the unilaterally symptomatic group, although the bilaterally symptomatic group did not show significant differences in this regard. As regards the disk positions in the joints of the three groups (asymptomatic group, unilaterally and bilaterally symptomatic groups), there were significant interactive effects of disk positions, regardless of gender. There were statistically significant age-related differences in disk deformities in all symptom groups, regardless of gender, except for in the group of males lacking symptoms in either joint. As regards disk deformities among the three groups studies here, there were significant interactive effects for disk positions, regardless of gender.
\end{abstract}

Key Words: TMD, MRI, Disk.

\section{INTRODUCTION}

Temporomandibular disorder (TMD) is a disease which causes wide-ranging and diverse clinical symptoms in the head and neck region. Headache, prosopalgia, movement disturbance, and joint noise develop in the temporomandibular joint (TMJ), masticatory musculature, and associated anatomical structures $[1,2]$. It is generally reported that the occurrence rate of TMD begins to increase in the 2nd decade of life, unlike other joint diseases in the human body [3,4]. Isberg et al. [4] reported from a study of 248 consecutive patients that there was a statistically significant peak in the development of TMJ disk displacement expressed with subsequent pain during adolescence. Katzberg et al. [5] reported that the incidence of joint noise increased in by approximately $17.5 \%$ for 2 years when the subjects were in their late teens. Howard [6] reported finding a majority of patients with TMD in the range of 15 to 45 years old. It has also been reported that approximately $25 \%$ of one sample had TMDrelated clinical symptoms [7]. Schifman et al. [8] reported the results of an epidemiological study of patients without

*Address correspondence to this author at the Department of Oral and Maxillofacial Radiology, Field of Tumor Biology, Okayama University Graduate School of Medicine and Dentistry, 5-1, Shikata-cho, 2-Chome, Okayama-city, Okayama 700-8525, Japan; Tel: +81-86-235-6706; Fax: +81-86235-6709; E-mail: asaumi@md.okayama-u.ac.jp symptoms of TMD, noting that approximately $75 \%$ of the subjects studied showed TMJ dysfunction manifestations such as mandibular motor abnormality, joint noise, and tenderness upon palpation; furthermore, approximately $33 \%$ of those subjects exhibited clinical symptoms such as arthralgia, prosopalgia, and a limited range for the opening of the mouth. Huber et al. [9] also reported that approximately 50\% of their sample showed joint noise or mandibular deviation upon opening the mouth, and approximately $5 \%$ of the subjects exhibited limited ability to open the mouth. Barclay et al. [10] revealed that over $30 \%$ of their study sample showed more than one TMD-related clinical symptom, and only approximately 5 to $7 \%$ of their subjects required treatment for TMD. Therefore, the diagnosis of TMD should be based on each patient's past history, as well as on the clinical and radiological manifestations.

As regards the association between a patient's clinical manifestations and the interpretation of MR images in the diagnosis of TMD, Margulles-Bonnet et al. [11] reported a congruity of approximately 60\%; Robert et al. [12] reported a congruity of ca. 58\%; and Paesani et al. [13] reported a congruity of ca. 43\%. Emshoff et al. [14] reported that pain in the TMJ region had no remarkable relationship to internal TMJ derangement diagnosed by MRI. Haley et al. [15] also reported finding no significant correlation between pain in 
the TMJ region and disk displacement identified using MRI. Moreover, Barclay et al. [10] demonstrated that the clinical diagnostic criteria can be an index of TMJ internal derangement, but that such criteria do not reveal the degree of disk displacement. In contrast, Tasaki et al. [16] showed a correlation between disk displacement and TMD; $18 \%$ of a symptomatic group and $70 \%$ of an asymptomatic group showed normal disk placement. Likewise, Larheim et al. [17] showed similar rates of $22 \%$ and $65 \%$, respectively. There remains much controversy surrounding the correlation between clinical and MRI manifestations in patients with TMD. Reports of TMD presenting as a lump in younger patients have provided only insufficient evidence, as have reports that TMD most often occurs among females. It has also been noted that MRI examination can produce varied results due to the variety of judgment criteria [14-21]. It is necessary to more accurately assess tentative clinical diagnoses when arriving at a treatment plan for patients with TMD. Moreover, it is considered to be very important to define the morphologic and functional changes in the anatomical structure of the TMJ region and to follow the progression of TMD based on the clinical manifestations observed upon MRI in patients with TMD.

So, we thought that disk position and disk deformity might be different because of the age and gender, and then that they might influence clinical symptoms. Thus, we conducted the present comparative observation of MRI manifestations according to gender and age in order to identify the correlation between clinical manifestations and MRI findings in a large sample of patients with TMD.

\section{MATERIAL AND METHODS}

We conducted a study of patients with TMD who had been screened by a retrospective evaluation of case histories. The evaluation included the cases of 1,500 consecutive patients who underwent MRI of the TMJ region at Okayama University Hospital of Dentistry at some point in time during the four years ranging from 1998 to 2001. All subjects gave their informed consent. The patients with symptoms of TMD were selected among those who underwent the appropriate clinical examination and registration. Patients were excluded who had undergone diagnostic examination for orthodontic treatment or orthopedic surgery that was not related to their chief complaint, those who were suspected of having a neoplasm (e.g., osteochondroma or synovial condromatosis, etc.) in the TMJ region, those who were suspected of having pain related to an infection in the jaws and/or salivary glands, those who suffered from headache (and were therefore difficult to differentially diagnose), and those who had a past history of trauma within three months prior to the examination. Several cases that were difficult to evaluate due to the low quality of the MR images were also excluded. MRI interpretation was performed in a confidential manner. Following the process of exclusion, a total of 1,265 patients were enrolled in the present study.

After the clinical evaluation and case history examination of the TMJ had been carried out, TMJ joints were classified as follows. The following joints were included in the asymptomatic group: those presenting without subjective symptoms; those which, according to the patient's self-report, had not previously required treatment or diagnosis due to joint noise, pain, or mouth-opening disturbances; and those lacking positive results upon examination for the present episode. The remaining TMJ patients were categorized either into the unilaterally symptomatic group (patients with unilateral symptoms in the TMJ) and the bilaterally symptomatic group (patients with bilateral symptoms in the TMJ). The unilateral symptom group was subdivided into two further groups, i.e., asymptomatic and unilaterally symptomatic joints; thus, 3 experimental groups were formed. Each experimental group was classified by gender, and then, each gender group was divided into seven stages from the 2nd decades of life (ages 10-19) to the 8th decades of life (ages 70-79). Each experimental group underwent MRI examination in order to observe disk positions and disk deformities.

\section{MRI Interpretation}

During MRI examination of both TMJs, the head was positioned with a Frankfort's line vertical to the table of the MRI device, thus acquiring oblique sagittal plan images vertical to each condylar angulation from the intercuspal position and the maximum opening position. The MR examinations were performed using a 1.5T unit (Magnetom vision; Siemens, Erlangen, Germany) with a TMJ surface coil. The following MRI scanning parameters were applied: fatsuppressed oblique sagittal images (double contrast turbospinecho [SE] method; repetition time [TR] ms/effective echo time [TE] $\mathrm{ms}=2400-2600 / 14,85 \mathrm{~ms}$, five echo train lengths, slice thickness; $3 \mathrm{~mm}$, field of view [FOV]; $125 \mathrm{~mm}$ x $125 \mathrm{~mm}$, pixel size; $0.78 \mathrm{~mm}$ x $0.49 \mathrm{~mm}$ ) at the open and closed mouth were acquired. According to the abovementioned provisions, seven slice of images were obtained for each unilateral joint and a multi-slice examination was carried out each of cases. Osiris 4.19 (University Hospitals of Geneva, Switzerland) from the freeware Dicom viewer software was used to evaluate the obtained images on a highresolution screen displayer for interpretation by two experienced and skilled oral and maxillofacial radiologists (Choi \& Yanagi). Interpretation was conducted according to predetermined criteria (see below for details), and conclusions were arrived at on a consensus basis in cases involving any disagreement between observers. The interpretation of specific features for observation was carried out according to the following criteria.

\section{Disk Position}

The intermediate zone criterion of disk displacement suggested by Orsini et al. [19] was used to determine disk displacement of the TMJ upon interpretation of the images of patients with TMD. A case meeting determined as normal in all slices was used as a control. Joints thought to exhibit disk displacement underwent angular measurement at the mesial, central, and lateral regions of each joint on the basis of the method of Rammelsberg et al. [20]. As regards angular measurement, image of each slice were evaluated to measure identical regions among all slices of the following 3 regions: the uppermost point of the borderline between the posterior band and the retrodiskal tissue, the central point of the thickest part of the posterior band, and the central point of the intermediate zone. Both evaluation and measurement were carried out on the oblique sagittal plane, and the following criteria were used for the classification of disk positions and for mouth closing positions. 
a) Normal superior position (NS): The intermediate zone of the disk was located between the articular surface of the condyle and the posterior slope of the articular eminence in all slices according to the intermediate zone criterion (Fig. 1a).

b) Partial disk displacement in the mesial region (PM): Disk displacement was observed in the image slice of the mesial region of the condyle according to the intermediate zone criterion, and the disk was normally positioned in the remaining image slices (Fig. 1b).

c) Partial disk displacement in the lateral region (PL): Disk displacement was observed in the slice of the lateral region of the condyle, according to the intermediate zone criterion, and the disk was positioned normally in the remaining slices (Fig. 1c).

d) Complete disk displacement with severe anterior displacement in the mesial region (CM): Anterior displacement was seen in all slices, according to the intermediate zone criterion, and the disk was located more than 15 degrees anterior to the lateral area with respect to the mesial region, based on angular measurements (Fig. 1d). e) Complete disk displacement with more anterior displacement in the lateral region (CL): Anterior displacement was seen in all slices according to the intermediate zone criterion, and the disk was located more than 15 degrees anterior to the mesial region in the lateral region based on angular measurement (Fig. 1e).

f) Complete disk displacement with severe anterior displacement in all regions (CA): Anterior displacement was seen in all slices according to the intermediate zone criterion, and the displacement was less than 15 degrees compared to the mesial region in the lateral region in all image slices. This criterion also included cases with severe anterior displacement of more than 70 degrees, observed in all slices, regardless of the difference between mesial and lateral structures (Fig. 1f).

\section{Disk Deformities}

Determination of disk deformities was based on images of the intercuspal area and images of the mouth opening, both of which were obtained using an assistant apparatus. An evaluation of disk configurations was carried out in order to

a)
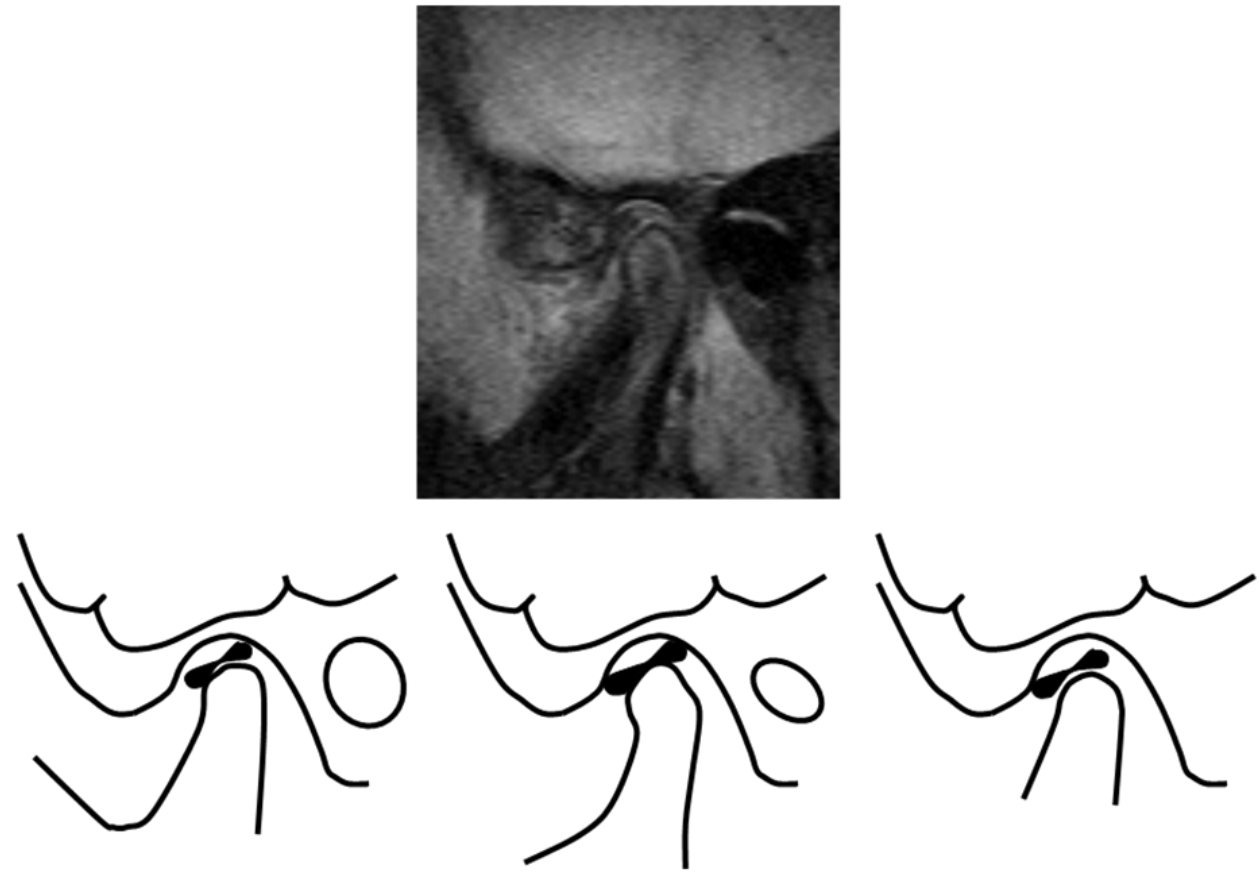

lateral region

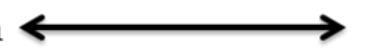

mesial region

b)
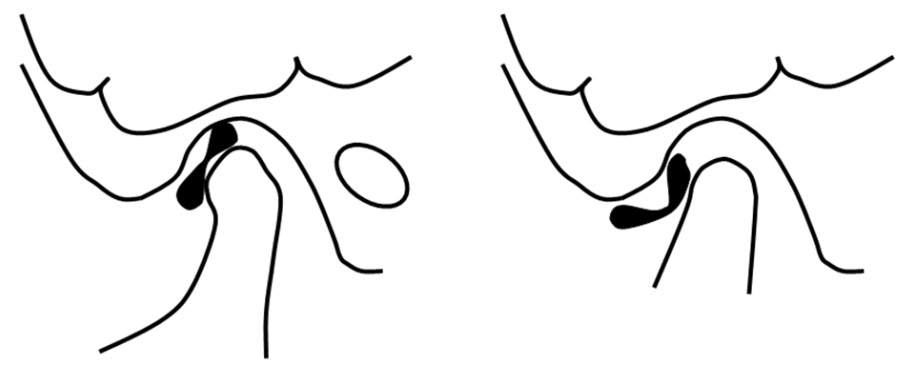
(Fig. 1) contd....

c)

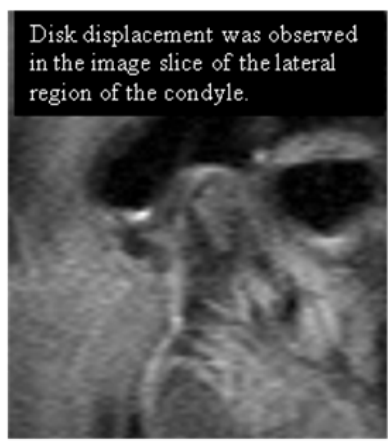

lateral region

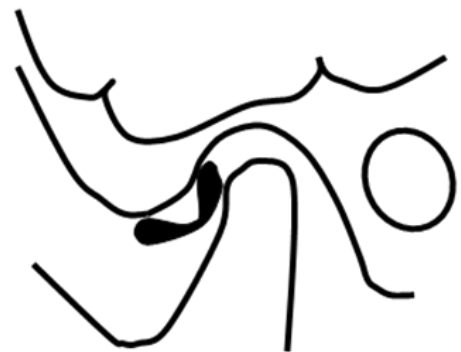

d)
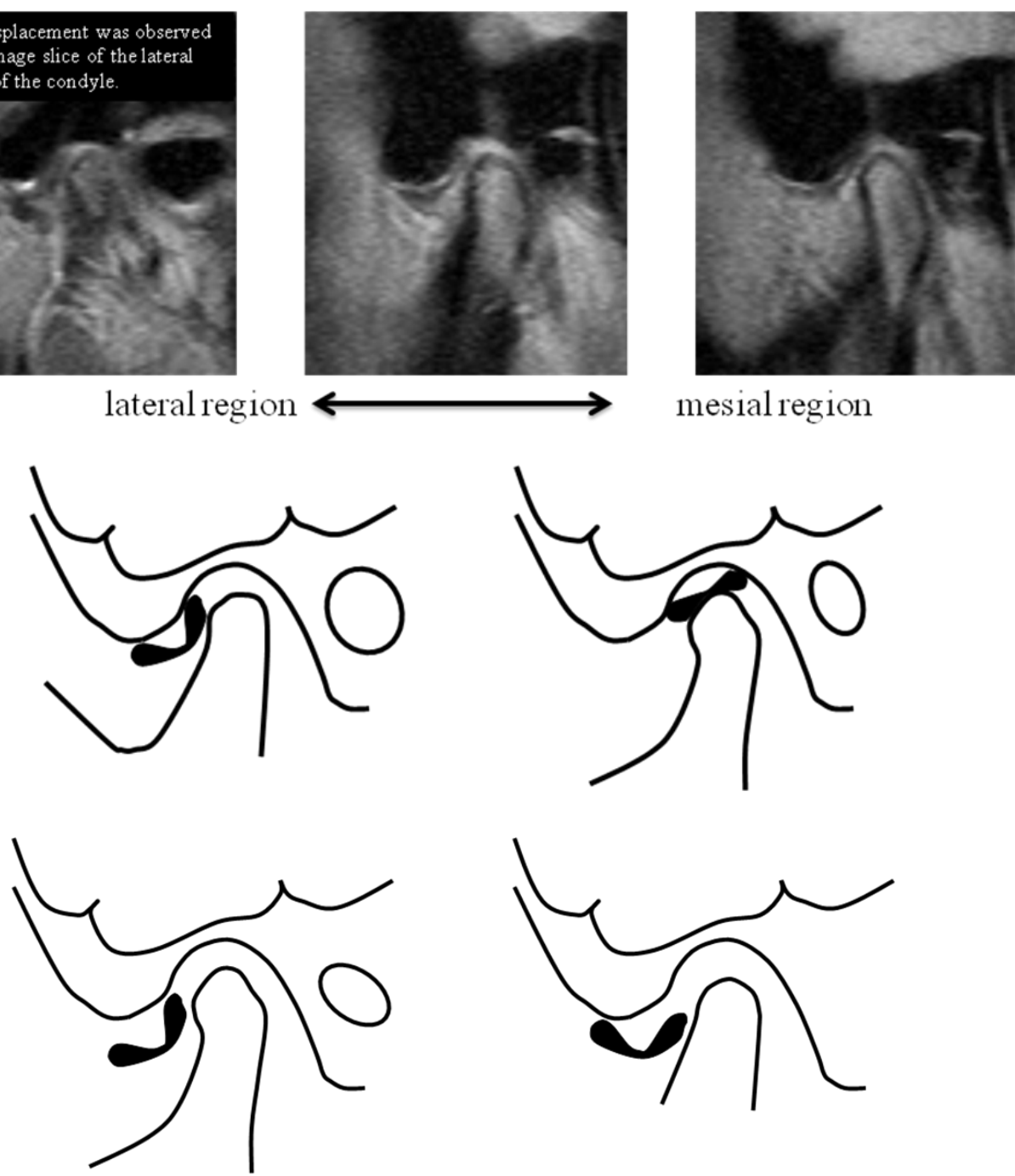

mesial region
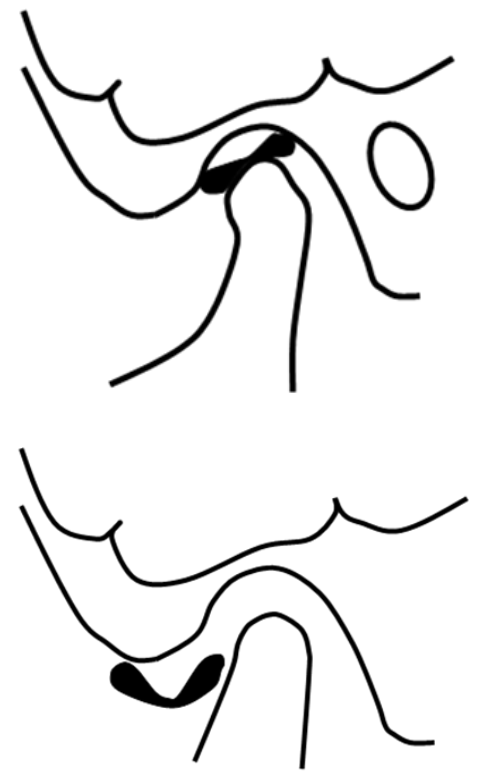

lateral region $\longleftrightarrow$ mesial region

e)
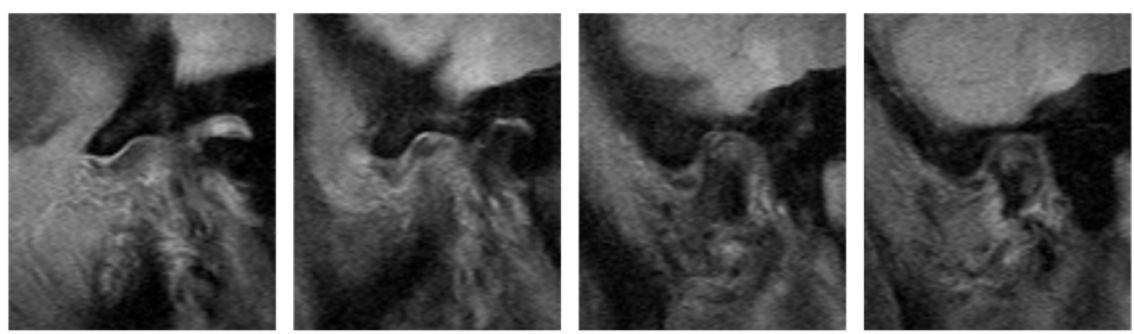

lateral region
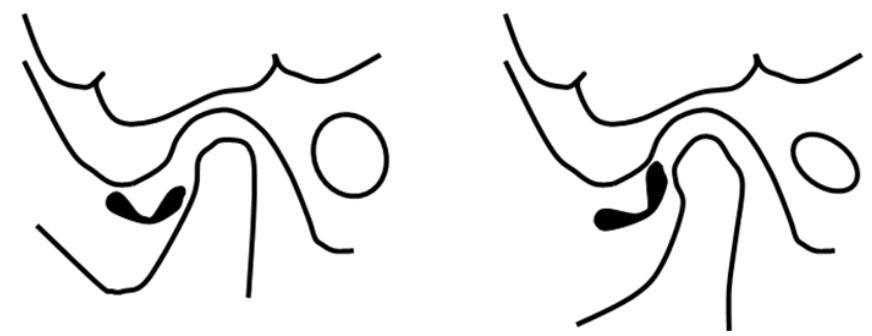
f)

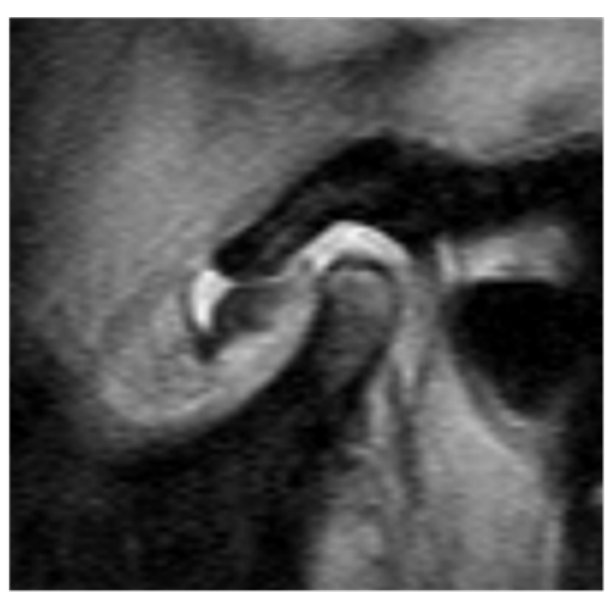

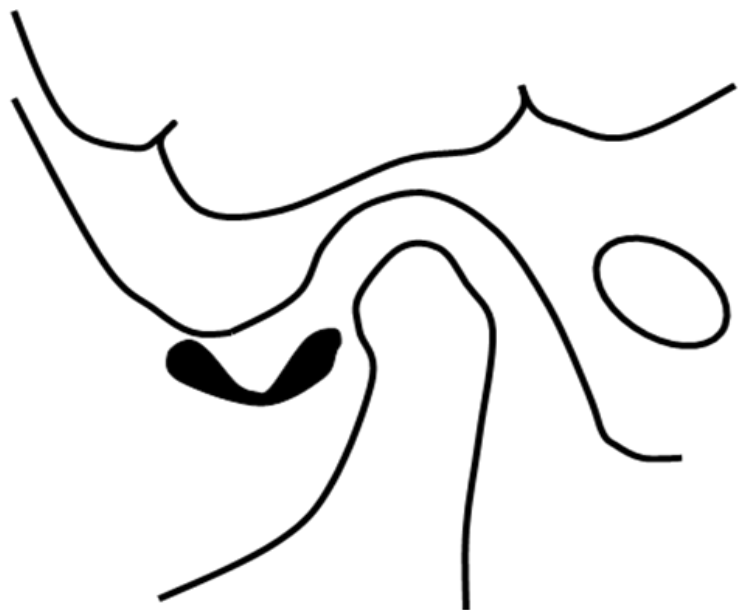

(Fig. 1) contd....

Fig. (1). a) Normal superior position (NS). b) Partial disk displacement in the mesial region (PM). c) Partial disk displacement in the lateral region (PL). d) Complete disk displacement with severe anterior displacement in the mesial region (CM). e) Complete disk displacement with more anterior displacement in the lateral region (CL). f) Complete disk displacement with severe anterior displacement in all regions (CA).

examine disk shape in regions with the most severe anterior displacement with the respect to the above-mentioned disk positions. The disk deformities were classified according to the following criteria.

a) Normal shape (NS): Disk in the normal superior position were included in this category; this type of disk was also included in the flatten disk (FL) category in cases involving uniform thickness without division into the anterior band, the intermediate zone, and the posterior band even during mouth opening.

b) Biconcave shape (BC): A displaced disk exhibiting a clear division of the anterior band, the intermediate zone, and the posterior band. The intermediate zone showed no or only slight shorting with respect to over a third of the full-length of the disk. Morphologically, the disk configuration maintained a bow-tie shape or showed some thickening of the posterior band.

c) Folded shape (FO): The displaced disk showed a clear division between the anterior band, the intermediate zone, and the posterior band. The intermediate zone exhibited no shortening by over a third of the full length determined as disk. Morphologically, the intermediate zone showed bending and assumed as cap or cup shape.

d) Biconvex shape (BV): The displaced disk showed no clear division between the anterior band and the anterior articular capsule; the intermediate zone was short. The posterior band adopted a roundish, convex shape. Cases showing retrodiscal tissue exhibited a convex shape with respect to the posterior band.

e) Flatten shape (FL): Normal-placed or displaced disks showing no divisions between the anterior band, the intermediate zone, and the posterior band; generally, this type exhibited uniform thinness, even when observed during mouth opening.

f) Amorphous shape: The disk assumed a shape such that it could not be evaluated according to the above-mentioned criteria. This category was also applied when the disk appeared to be fragment.

\section{Disk Recapture During Mouth Opening}

a) Disk displacement with reduction (WR): The intermediate zone of the displaced disk during mouth closing was restored to the position between the articular eminence and articular surface of the condyle during mouth opening.

b) Disk displacement without reduction (WoR): The intermediate zone of the displaced disk during mouth closing was not restored to the position between the articular eminence and the articular surface of the condyle during mouth opening.

\section{Statistical Analysis}

A chi-square $\left(\mathrm{X}^{2}\right)$ analysis was performed in order to test differences in the frequency of disk positions and disk deformities according to age and gender within the appropriate experimental group and to identify differences in disk deformities according to disk position in each experimental group. Two-way ANOVA was carried out to identify differences in the rate of manifestation of particular disk positions and disk deformities among asymptomatic joints, unilaterally symptomatic joints, and bilaterally symptomatic joints among males, females, and all patients. The empirical analyses were all tested at a significance level of $\mathrm{p}<0.05$, and statistical processing was carried out using the SPSSWIN 11.0 program.

\section{RESULTS}

The patients included of 946 females $(74.8 \%$; average, 36.6 years old), and 319 males ( $25.2 \%$; average, 34.3 years old). Both males and females in their 2nd decade of life showed the highest distribution of $31.4 \%$ and $31.2 \%$, respectively. Of all TMD patients, 945 (74.7\%) showed symptoms in the unilateral TMJ, and $320(25.3 \%)$ had symptoms in the bilateral TMJ (Table 1, Fig. 2).

\section{Disk Position in the Asymptomatic Joint Group Accord- ing to Gender and Age (Table 2)}

Among females, NS (57\%) had the highest frequency, followed by CA $(21 \%)$ and PL (10\%); however, there was 
Table 1. The Population of TMD Patients to be Participated in this Study

\begin{tabular}{|c|c|c|c|c|c|c|c|c|c|c|c|c|c|c|c|c|c|}
\hline \multirow{2}{*}{$\begin{array}{l}\text { Age } \\
\text { Sex }\end{array}$} & \multicolumn{2}{|c|}{ 2nd } & \multicolumn{2}{|c|}{ 3rd } & \multicolumn{2}{|c|}{ 4th } & \multicolumn{2}{|c|}{ 5th } & \multicolumn{2}{|c|}{ 6th } & \multicolumn{2}{|c|}{7 th } & \multicolumn{2}{|c|}{ 8th } & \multicolumn{2}{|c|}{ Sum } & \multirow{2}{*}{$\begin{array}{c}\text { Total } \\
\mathbf{F}+\mathbf{M}\end{array}$} \\
\hline & $\mathbf{F}$ & M & $\mathbf{F}$ & $\mathbf{M}$ & $\mathbf{F}$ & $\mathbf{M}$ & $\mathbf{F}$ & $\mathbf{M}$ & $\mathbf{F}$ & M & $\mathbf{F}$ & M & $\mathbf{F}$ & $\mathbf{M}$ & $\mathbf{F}$ & $\mathbf{M}$ & \\
\hline No. of Pt. & 145 & 71 & 295 & 100 & 142 & 48 & 106 & 31 & 137 & 28 & 79 & 27 & 42 & 14 & 946 & 319 & 1265 \\
\hline Average & 16 & 16.7 & 24.2 & 24.1 & 33.7 & 34.1 & 44.6 & 42.1 & 54.1 & 54.6 & 63.8 & 64.9 & 74.7 & 74 & 36.6 & 34.3 & 36 \\
\hline s.d. & 2.3 & 1.9 & 2.9 & 2.7 & 2.8 & 2.8 & 2.8 & 2.9 & 2.7 & 3 & 2.8 & 3.1 & 3.9 & 3.3 & 17.4 & 17.4 & 17.4 \\
\hline uni-lateral & 107 & 55 & 203 & 68 & 108 & 41 & 74 & 23 & 104 & 21 & 67 & 27 & 34 & 13 & 697 & 248 & 945 \\
\hline bi-lateral & 38 & 16 & 92 & 32 & 34 & 7 & 32 & 8 & 33 & 7 & 12 & 0 & 8 & 1 & 249 & 71 & 320 \\
\hline
\end{tabular}

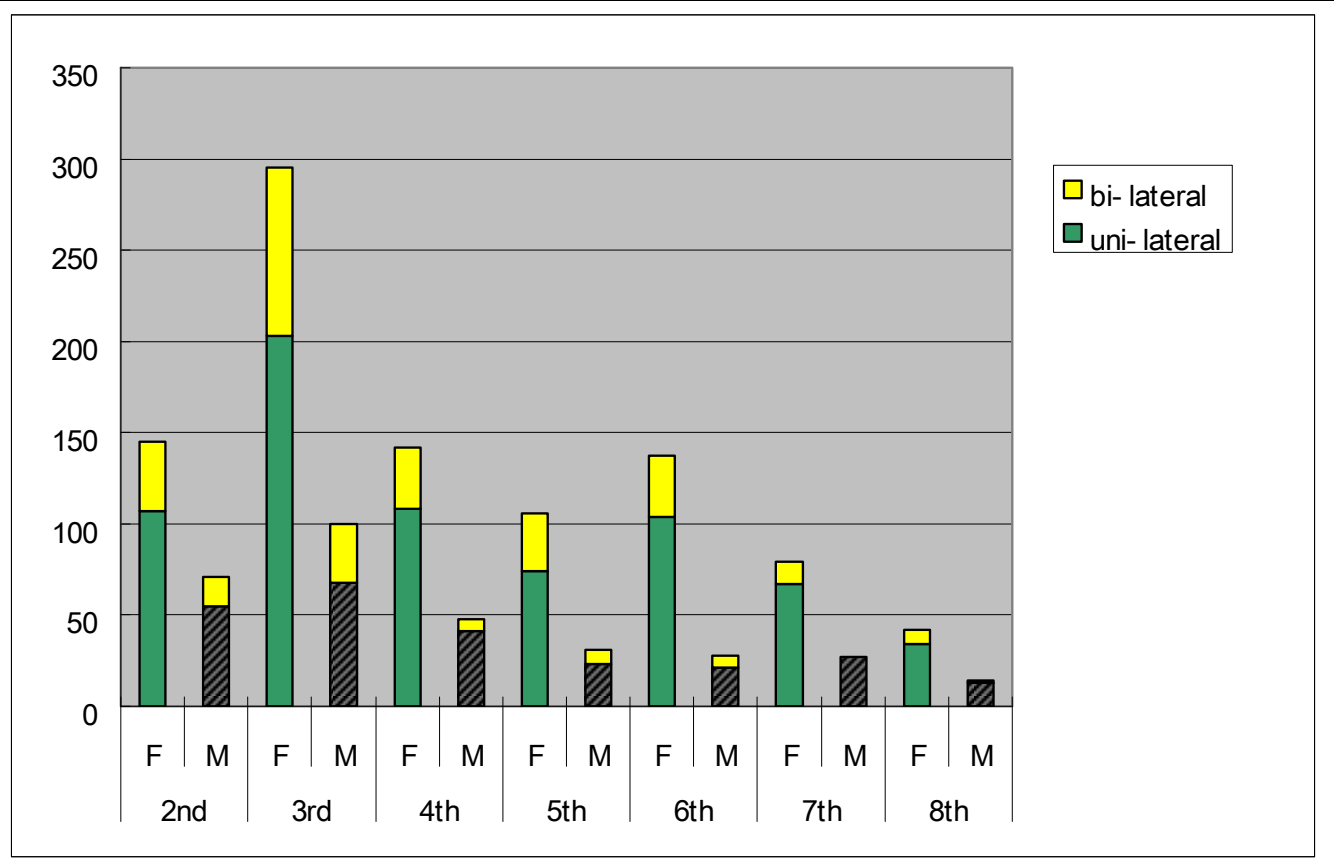

Fig. (2). The population of TMD patients to be participated in this study.

no significant difference with respect to age $(\mathrm{p}>0.05)$. Among the males, NS (69\%) showed the highest frequency, followed by CA (16\%) and PL (7\%); however, there was no significant difference with respect to age $(\mathrm{p}>0.05)$. Among all of the patients, NS (60\%) was the most frequency observed. As regards disk position, NS (50\%) showed the lowest frequency in the 5 th decade of life, but this result did not reveal a significant difference with respect to the frequency of disk positions according to age $(p>0.05)$. Significant differences in the distribution of disk position between males and females were observed, i.e., rates of $69 \%$ and $57 \%$ were observed in the NS group, $16 \%$ and $21 \%$ in the CA group, and $3.2 \%$ and $6.9 \%$ in the CL group, respectively $(\mathrm{p}<0.05)$.

\section{Disk Positions in Unilaterally Symptomatic Joints According to Gender and Age (Table 3)}

Among females, CA (40\%) showed the highest frequency, followed by CL (26\%) and NS (21\%). With respect to disk position, a higher frequency of CL (40\%), but a lower frequency of NS (10\%) was observed in the 2nd decade of life, in comparison with the other age groups. Patients in their 8th decade showed a high frequency of CA (56\%), followed by those in the 4th decade and 6th decade, which were both at the $46 \%$ level. Patients in their 7 th decade showed a high frequency of NS $(30 \%)$, followed by patients in the 3 rd decade $(26 \%)$. There were statistically significant differences in terms of the distribution of disk position according to age in females $(\mathrm{p}<0.05)$. In males, NS $(48 \%)$ was the most frequency observed, followed by CA (24\%) and CL (16\%). As regards disk position in the 2 nd decade of life, higher frequency of CL $(36 \%)$ and CA $(31 \%)$ were observed, but a lower frequency of NS (22\%) was observed than in the other age groups. Patients in their 5th and 8th decades showed a higher frequency of NS, i.e., $74 \%$ and $77 \%$, respectively, than did those in the other age groups. There were statistically significant differences in terms of the distribution of disk position according to age among males $(\mathrm{p}<0.01)$. In the group including all patients, CA $(36 \%)$ was the most frequency observed, followed by NS (28\%) and CL (23\%). The disk positions in patients in their 2nd decade showed a higher frequency of CL (39\%), but a lower frequency of NS $(14 \%)$ than those in the other age groups. The patients in their 8 th decade showed the highest frequency of CA $(45 \%)$, followed by those in their 4th decade and 7th decade, i.e., 
Table 2. Disk Positions for no Symptom Joints According to Sex and Age

\begin{tabular}{|c|c|c|c|c|c|c|c|c|c|c|c|c|c|c|c|c|}
\hline \multicolumn{17}{|c|}{ No-Symptom Joints } \\
\hline \multirow{2}{*}{ Age } & \multirow{2}{*}{ Sex } & \multicolumn{12}{|c|}{ Disk Positions } & \multirow{2}{*}{ Sum } & \multirow{2}{*}{$\%$} & \multirow{2}{*}{$X^{2}(\mathbf{P})$} \\
\hline & & NS & $\%$ & PM & $\%$ & PL & $\%$ & CM & $\%$ & $\mathbf{C L}$ & $\%$ & CA & $\%$ & & & \\
\hline \multirow{3}{*}{ 2nd } & $\mathrm{F}$ & 64 & 59.8 & 4 & 3.7 & 11 & 10.3 & 2 & 1.9 & 6 & 5.6 & 20 & 18.7 & 107 & 100 & \multirow{21}{*}{$\begin{array}{c}\text { female }(\mathrm{F}) \\
28.910 \\
(.522) \\
(\mathrm{p}>.05) \\
\text { male }(\mathrm{M}) \\
18.914 \\
(.757) \\
(\mathrm{p}>.05) \\
\mathrm{F}+\mathrm{M} \\
31.443 \\
(.394) \\
(\mathrm{p}>.05)\end{array}$} \\
\hline & M & 31 & 56.4 & 5 & 9.1 & 5 & 9.1 & 0 & 0 & 4 & 7.3 & 10 & 18.2 & 55 & 100 & \\
\hline & $\mathrm{F}+\mathrm{M}$ & 95 & 58.6 & 9 & 5.6 & 16 & 9.9 & 2 & 1.2 & 10 & 6.2 & 30 & 18.5 & 162 & 100 & \\
\hline \multirow{3}{*}{ 3rd } & $\mathrm{F}$ & 115 & 56.7 & 8 & 3.9 & 19 & 9.4 & 1 & 0.5 & 14 & 6.9 & 46 & 22.7 & 203 & 100 & \\
\hline & M & 48 & 70.6 & 3 & 4.4 & 3 & 4.4 & 0 & 0 & 2 & 2.9 & 12 & 17.6 & 68 & 100 & \\
\hline & $\mathrm{F}+\mathrm{M}$ & 163 & 60.1 & 11 & 4.1 & 22 & 8.1 & 1 & 0.4 & 16 & 5.9 & 58 & 21.4 & 271 & 100 & \\
\hline \multirow{3}{*}{ 4th } & $\mathrm{F}$ & 48 & 44.4 & 4 & 3.7 & 13 & 12 & 3 & 2.8 & 14 & 13 & 26 & 24.1 & 108 & 100 & \\
\hline & $\mathrm{M}$ & 27 & 65.9 & 2 & 4.9 & 4 & 4.9 & 0 & 0 & 1 & 2.4 & 7 & 17.1 & 41 & 100 & \\
\hline & $\mathrm{F}+\mathrm{M}$ & 75 & 50.3 & 6 & 4 & 17 & 11.4 & 3 & 2 & 15 & 10.1 & 33 & 22.1 & 149 & 100 & \\
\hline \multirow{3}{*}{ 5th } & $\mathrm{F}$ & 43 & 58.1 & 2 & 2.7 & 5 & 6.8 & 0 & 0 & 4 & 5.4 & 20 & 27 & 74 & 100 & \\
\hline & M & 20 & 87 & 0 & 0 & 0 & 0 & 0 & 0 & 1 & 4.3 & 2 & 8.7 & 23 & 100 & \\
\hline & $\mathrm{F}+\mathrm{M}$ & 63 & 64.9 & 2 & 2.1 & 5 & 5.2 & 0 & 0 & 5 & 5.2 & 22 & 22.7 & 97 & 100 & \\
\hline \multirow{3}{*}{ 6th } & $\mathrm{F}$ & 64 & 61.5 & 4 & 3.8 & 13 & 12.5 & 0 & 0 & 7 & 6.7 & 16 & 15.4 & 104 & 100 & \\
\hline & $\mathrm{M}$ & 14 & 66.7 & 1 & 4.8 & 3 & 14.3 & 0 & 0 & 0 & 0 & 3 & 14.3 & 21 & 100 & \\
\hline & $\mathrm{F}+\mathrm{M}$ & 78 & 62.4 & 5 & 4 & 16 & 12.8 & 0 & 0 & 7 & 5.6 & 19 & 15.2 & 125 & 100 & \\
\hline \multirow{3}{*}{ 7th } & $\mathrm{F}$ & 43 & 64.2 & 2 & 3 & 6 & 9 & 2 & 3 & 3 & 4.5 & 11 & 16.4 & 67 & 100 & \\
\hline & M & 21 & 77.8 & 1 & 3.7 & 1 & 3.7 & 0 & 0 & 0 & 0 & 4 & 14.8 & 27 & 100 & \\
\hline & $\mathrm{F}+\mathrm{M}$ & 64 & 68.1 & 3 & 3.2 & 7 & 7.4 & 2 & 2.1 & 3 & 3.2 & 15 & 16 & 94 & 100 & \\
\hline \multirow{3}{*}{ 8th } & $\mathrm{F}$ & 22 & 64.7 & 1 & 2.9 & 2 & 5.9 & 1 & 2.9 & 0 & 0 & 8 & 23.5 & 34 & 100 & \\
\hline & $\mathrm{M}$ & 11 & 84.6 & 0 & 0 & 1 & 7.7 & 0 & 0 & 0 & 0 & 1 & 7.7 & 13 & 100 & \\
\hline & $\mathrm{F}+\mathrm{M}$ & 33 & 70.2 & 1 & 2.1 & 3 & 6.4 & 1 & 2.1 & 0 & 0 & 9 & 19.1 & 47 & 100 & \\
\hline \multirow{3}{*}{ sum } & $\mathrm{F}$ & 399 & 57.2 & 25 & 3.6 & 69 & 9.9 & 9 & 1.3 & 48 & 6.9 & 147 & 21.1 & 697 & 100 & \multirow{3}{*}{$\begin{array}{l}\mathrm{P}=.004 \\
(\mathrm{P}<.01)\end{array}$} \\
\hline & M & 172 & 69.4 & 12 & 4.8 & 17 & 6.9 & 0 & 0 & 8 & 3.2 & 39 & 15.7 & 248 & 100 & \\
\hline & $\mathrm{F}+\mathrm{M}$ & 571 & 60.4 & 37 & 3.9 & 86 & 9.1 & 9 & 1 & 56 & 5.9 & 186 & 19.7 & 945 & 100 & \\
\hline
\end{tabular}

both of the latter were at the $42 \%$ level. Those in their 7 th and 8th decades showed a higher frequency of NS, i.e., $40 \%$ and $38 \%$, respectively, than those in the other age groups. There were statistically significant differences in terms of the distribution of disk position according to age among all patients $(\mathrm{p}<0.001)$. As regards the distribution of disk positions between males and females, the following significant differences were observed: $48 \%$ and $21 \%$ in the NS group, $24 \%$ and $40 \%$ in the CA group, and $16 \%$ and $26 \%$ in the CL group, respectively $(\mathrm{p}<0.001)$.

Disk Positions in Bilaterally Symptomatic Joints According to Gender and Age (Table 4)

Among females, NS (34\%) was the most frequently observed group, followed by CA (30\%) and CL (19\%). As re- gards disk position, patients in their 2nd decade showed a higher frequency of CA $(34 \%)$ and CL $(28 \%)$, but a lower frequency of NS $(21 \%)$ than did the patients in the other age groups. Patients in their 8th and 7the decades showed high frequencies of CA, $56 \%$ and $42 \%$, respectively. Those in their 6 th and 7 th decades showed high frequencies of NS, $46 \%$ and $42 \%$, respectively. There were statistically significant differences in terms of disk position frequency according to age in females $(\mathrm{p}<0.05)$. Among males, NS $(46 \%)$ was the most frequently observed group, followed by CA $(28 \%)$ and CL (14\%). As regards disk position in the 2nd decade of life, a higher frequency of CA (53\%), but lower frequency of NS $(22 \%)$ was observed than in the other age groups. The older the patients were, the higher was frequency of NS. Statistically significant differences were observed among males 
Table 3. Disk Positions for Uni-Lateral Symptoms Joints According to Sex and Age

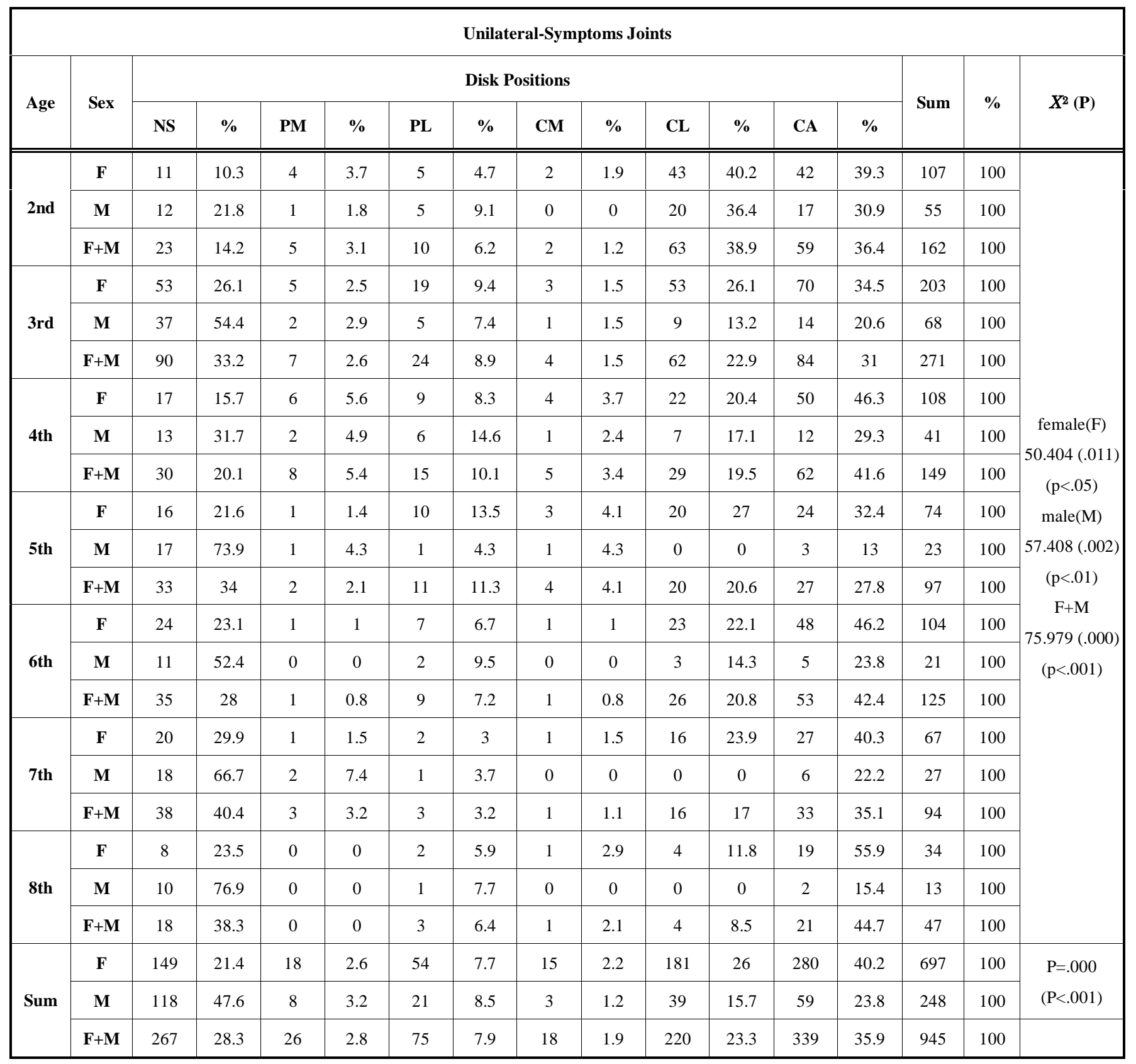

terms of the frequency of disk position according to age $(\mathrm{p}<0.05)$. In all of the patients, NS $(37 \%)$ was the most frequently observed, followed by CA $(30 \%)$ and CL (18\%). Also with respect to disk position, a higher frequency of $\mathrm{CA}$ (40\%) and CL (25\%), but a lower frequency of NS $(21 \%)$ was observed among teens than those in the other age groups. Those in their 8 th and 7 th decades showed a high frequency of CA, i.e., $50 \%$ and $42 \%$, respectively. Those in their 6th and 5th decades showed a higher frequency of NS, i.e., $48 \%$ and $41 \%$, respectively, than those in the other age groups. There were statistically significant differences in the frequency of disk positions according to age in the group in which all patients were considered together $(\mathrm{p}<0.001)$. As regards the distribution of disk position in males and females, no significant difference were observed, i.e., $46 \%$ and
$34 \%$ in the NS group, $28 \%$ and $30 \%$ in the CA group, and $14 \%$ and $19 \%$ in the CL group, respectively ( $>0.05)$.

Disk Positions in Three Experimental Groups; Asymptomatic, and Unilaterally, and Bilaterally Symptomatic Joint Groups (Two Way ANOVA Analysis)

As regards disk position among asymptomatic participants, and the unilaterally, and bilaterally symptomatic males, females, and total patient sample, there were significant interactive effects of disk positions according to groups $(p<0.001)$. These results demonstrated significant differences in terms of frequency of disk positions among all three groups, i.e., the asymptomatic group and the unilaterally and bilaterally symptomatic groups. 
Table 4. Disk Positions for Bi-Lateral Symptoms Joints According to Sex and Age

\begin{tabular}{|c|c|c|c|c|c|c|c|c|c|c|c|c|c|c|c|c|}
\hline \multicolumn{17}{|c|}{ bilateral-Symptoms Joints } \\
\hline \multirow{2}{*}{ Age } & \multirow{2}{*}{ Sex } & \multicolumn{12}{|c|}{ Disk Positions } & \multirow{2}{*}{ Sum } & \multirow{2}{*}{$\%$} & \multirow{2}{*}{$X^{2}(\mathbf{P})$} \\
\hline & & NS & $\%$ & PM & $\%$ & PL & $\%$ & $\mathbf{C M}$ & $\%$ & $\mathbf{C L}$ & $\%$ & CA & $\%$ & & & \\
\hline \multirow{3}{*}{ 2nd } & $\mathbf{F}$ & 16 & 21.1 & 3 & 3.9 & 7 & 9.2 & 3 & 3.9 & 21 & 27.6 & 26 & 34.2 & 76 & 100 & \multirow{21}{*}{$\begin{array}{c}\text { female }(\mathrm{F}) \\
50.382 \\
(.011) \\
(\mathrm{p}<.05) \\
\text { male }(\mathrm{M}) \\
39.842 \\
(.030) \\
(\mathrm{p}<.05) \\
\mathrm{F}+\mathrm{M} \\
59.951 \\
(.001) \\
(\mathrm{p}<.01)\end{array}$} \\
\hline & $\mathbf{M}$ & 7 & 21.9 & 0 & 0 & 2 & 6.3 & 0 & 0 & 6 & 18.8 & 17 & 53.1 & 32 & 100 & \\
\hline & $\mathbf{F}+\mathbf{M}$ & 23 & 21.3 & 3 & 2.8 & 9 & 8.3 & 3 & 2.8 & 27 & 25 & 43 & 39.8 & 108 & 100 & \\
\hline \multirow{3}{*}{ 3rd } & $\mathbf{F}$ & 63 & 34.2 & 12 & 6.5 & 14 & 7.6 & 5 & 2.7 & 39 & 21.2 & 51 & 27.7 & 184 & 100 & \\
\hline & $\mathbf{M}$ & 31 & 48.4 & 4 & 6.3 & 4 & 6.3 & 0 & 0 & 7 & 10.9 & 18 & 28.1 & 64 & 100 & \\
\hline & $\mathbf{F}+\mathbf{M}$ & 94 & 37.9 & 16 & 6.5 & 18 & 7.3 & 5 & 2 & 46 & 18.5 & 69 & 27.8 & 82 & 100 & \\
\hline \multirow{3}{*}{ 4th } & $\mathbf{F}$ & 22 & 32.4 & 2 & 2.9 & 10 & 14.7 & 6 & 8.8 & 13 & 19.1 & 15 & 22.1 & 68 & 100 & \\
\hline & $\mathbf{M}$ & 7 & 50 & 2 & 4.3 & 2 & 14.3 & 0 & 0 & 2 & 14.3 & 1 & 7.1 & 14 & 100 & \\
\hline & $\mathbf{F}+\mathbf{M}$ & 29 & 35.4 & 4 & 4.9 & 12 & 14.6 & 6 & 7.3 & 15 & 18.3 & 16 & 19.5 & 80 & 100 & \\
\hline \multirow{3}{*}{5 th } & $\mathbf{F}$ & 23 & 35.9 & 0 & 0 & 9 & 14.1 & 0 & 0 & 9 & 14.1 & 23 & 35.9 & 64 & 100 & \\
\hline & $\mathbf{M}$ & 10 & 62.5 & 0 & 0 & 2 & 12.5 & 0 & 0 & 2 & 12.5 & 2 & 12.5 & 16 & 100 & \\
\hline & $\mathbf{F}+\mathbf{M}$ & 33 & 41.3 & 0 & 0 & 11 & 13.8 & 0 & 0 & 11 & 13.8 & 25 & 31.3 & 80 & 100 & \\
\hline \multirow{3}{*}{ 6th } & $\mathbf{F}$ & 30 & 45.5 & 1 & 1.5 & 9 & 13.6 & 0 & 0 & 10 & 15.2 & 16 & 24.2 & 66 & 100 & \\
\hline & $\mathbf{M}$ & 8 & 57.1 & 0 & 0 & 1 & 7.1 & 1 & 7.1 & 3 & 21.4 & 1 & 7.1 & 14 & 100 & \\
\hline & $\mathbf{F}+\mathbf{M}$ & 38 & 47.5 & 1 & 1.3 & 10 & 12.5 & 1 & 1.3 & 13 & 16.3 & 17 & 21.3 & 80 & 100 & \\
\hline \multirow{3}{*}{ 7th } & $\mathbf{F}$ & 10 & 41.7 & 0 & 0 & 1 & 4.2 & 1 & 4.2 & 2 & 8.3 & 10 & 41.7 & 24 & 100 & \\
\hline & $\mathbf{M}$ & 0 & 0 & 0 & 0 & 0 & 0 & 0 & 0 & 0 & 0 & 0 & 0 & 0 & 100 & \\
\hline & $\mathbf{F}+\mathbf{M}$ & 10 & 41.7 & 0 & 0 & 1 & 4.2 & 1 & 4.2 & 2 & 8.3 & 10 & 41.7 & 24 & 100 & \\
\hline \multirow{3}{*}{ 8th } & $\mathbf{F}$ & 5 & 31.3 & 0 & 0 & 0 & 0 & 1 & 6.3 & 1 & 6.3 & 9 & 56.3 & 16 & 100 & \\
\hline & $\mathbf{M}$ & 2 & 100 & 0 & 0 & 0 & 0 & 0 & 0 & 0 & 0 & 0 & 0 & 2 & 100 & \\
\hline & $\mathbf{F}+\mathbf{M}$ & 7 & 38.9 & 0 & 0 & 0 & 0 & 1 & 5.6 & 1 & 5.6 & 9 & 50 & 18 & 100 & \\
\hline \multirow{3}{*}{ Sum } & $\mathbf{F}$ & 169 & 33.9 & 18 & 3.6 & 50 & 10 & 16 & 3.2 & 95 & 19.1 & 150 & 30.1 & 498 & 100 & \multirow{2}{*}{$\begin{array}{l}\mathrm{p}=.095 \\
(\mathrm{p}>.05)\end{array}$} \\
\hline & $\mathbf{M}$ & 65 & 45.8 & 6 & 4.2 & 11 & 7.7 & 1 & 0.7 & 20 & 14.1 & 39 & 27.5 & 142 & 100 & \\
\hline & $\mathbf{F}+\mathbf{M}$ & 234 & 36.6 & 24 & 3.8 & 61 & 9.5 & 17 & 2.7 & 115 & 18 & 189 & 29.5 & 640 & 100 & \\
\hline
\end{tabular}

Disk Deformities in Asymptomatic Joints According to Gender and Age (Table 5)

In females, NS (57\%) was most commonly observed, followed by BC (19\%) and BV (13\%). The disk deformities in study participants in the 4th decades of life (44\%) were associated with the lowest frequency of NS, whereas participants in their 7 th decade $(65 \%)$ showed the highest frequency of disk deformity. Subjects in their 4th decade $(23 \%)$ showed the highest frequency of BV, whereas the lower and higher age groups tended to exhibit decreased frequency in this regard. Whereas the frequency of FO tended to be inversely proportional to age, that of FL and AM tended to be proportional to age. There were statistically significant differences in terms of the frequency of disk deformity according to age in females $(p<0.001)$. In males, NS $(69 \%)$ was most commonly observed, followed by BC (17\%) and BV (5\%); however, no significant difference in the frequency of disk deformity was found to be associated with age ( $>0.05)$. When all patients were considered together, NS (60\%) was most frequently seen, followed by BC (18\%) and BV (11\%). Disk deformities in their 7 th and 8th decades showed a higher association with NS, i.e., $68 \%$ and $70 \%$, respectively, in comparison with the disk deformities in other age groups. Subjects in their 2nd decade (24\%) showed a higher frequency of BC, whereas those in 3rd (19\%) and 4th decades $(20 \%)$ showed a higher frequency of BV than did those in the other age groups. There were statistically significant differences in terms of the frequency of disk deformity according to age in the total patient sample $(\mathrm{p}<0.001)$. As regards the distribution of disk deformity among males and females, 
Table 5. Disk Deformities for No Symptom Joints According to Sex and Age

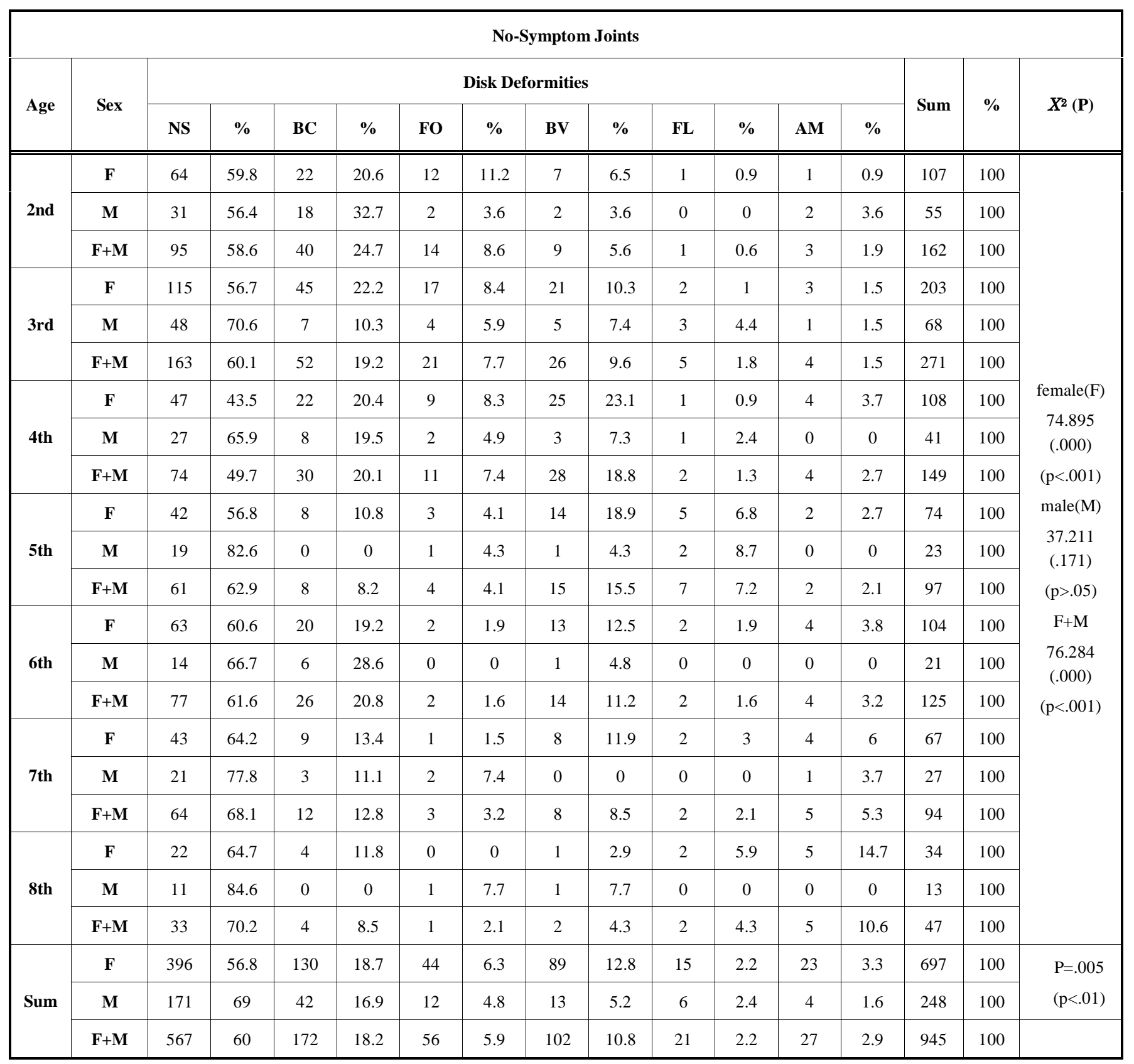

significant differences were observed, i.e., $69 \%$ and $57 \%$ in the NS group, $17 \%$ and $19 \%$ in the BC group, and $5 \%$ and $13 \%$ in the BV group, respectively $(\mathrm{p}<0.01)$.

\section{Disk Deformity in the Unilaterally Symptomatic Joints According to Gender and Age (Table 6)}

In females, BV (25\%) was most commonly seen, followed by BC (24\%), FO (23\%), and NS (21\%). The disk deformities in subjects in the 2nd decade of life showed a higher frequency of BC (34\%) and FO (36\%), and a lower frequency of NS $(10 \%)$, than was observed in the other age groups. BV was the most frequently seen among subjects in their 6th decade $(38 \%)$, and was least often seen among those in their 2 nd decade of life (16\%). Although no AM was observed among those in their 2nd and 3rd decades, the frequency of AM tended to be proportional to age. There were statistically significant differences in terms of the frequency of disk deformity according to age among females $(\mathrm{p}<0.001)$. In males, NS $(48 \%)$ was most frequently seen, followed by $\mathrm{BC}(21 \%)$ and FO (18\%). As regards disk deformities in subjects in their 5th and 8th decades, a higher frequency of NS, i.e., $74 \%$ and $77 \%$, respectively, was observed than in the other age groups, whereas subjects in their 2nd decade $(22 \%)$ showed the lowest frequency of disk deformity. FO was the most frequent among those in their 2nd decade (46\%), whereas BV was most commonly seen among those in their 4th decades $(25 \%)$. There were statistically significant differences among females in terms of the frequency of disk deformity in association with age $(\mathrm{p}<0.001)$. 
Table 6. Disk Deformities for Uni-Lateral Symptoms Joints by Sex and Age

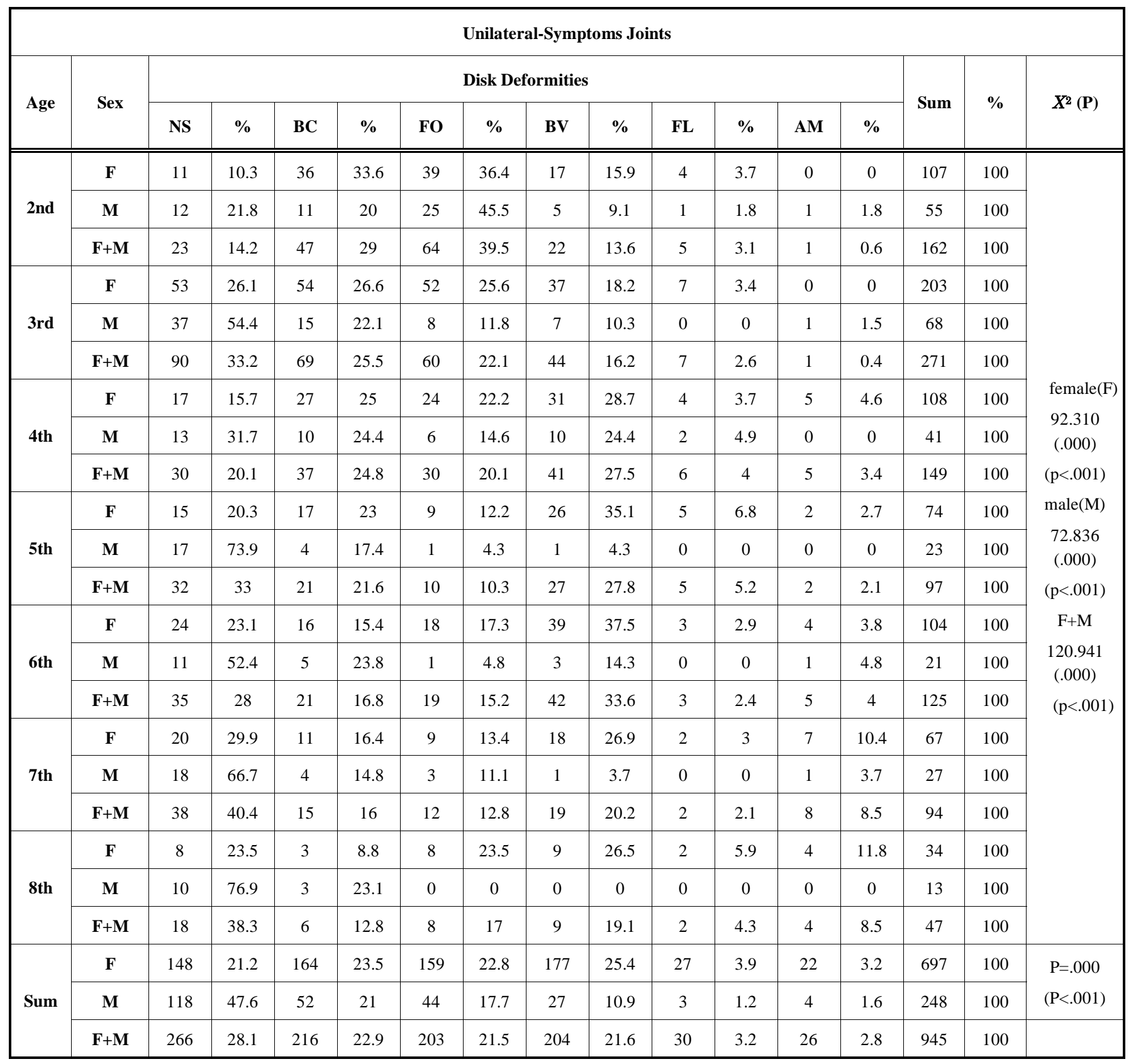

When all of the patients were considered together, NS (28\%) was the most frequently observed, followed by BC $(23 \%)$ and FO (22\%) and BV (22\%). As regards disk deformity among subjects in their 7th and 8th decades, a higher frequency of NS, $40 \%$ and $38 \%$, respectively, was observed than was observed in the other age groups, whereas subjects in their teens $(14 \%)$ showed the lowest frequency of NS. Subject in their 2nd decade of life showed a higher frequency of BC (29\%), while those in their 6th decade showed a higher frequency of BV (34\%) than did those in the other age groups. Subjects in their 2 nd decade (40\%) showed a remarkably higher frequency of FO than did the other age groups. When all patients were considered together, statistically significant differences were observed in terms of the frequency of disk deformity according to age $(p<0.001)$. As regards the distribution of disk deformities among males and females, significant differences were observed, i.e., $48 \%$ and $21 \%$ in the NS group, $21 \%$ and $24 \%$ in the BC group, and $11 \%$ and $26 \%$ in the BV group, respectively ( $<<0.001)$.

\section{Disk Deformity in Bilaterally Symptomatic Joints According to Gender and Age (Table 7)}

Among females, NS (32\%) was most frequently observed, followed BC (23\%), BV (21\%), and FO (15\%). As regards disk deformity assessed in terms of age, NS was most frequently observed among those in their 6th and 7th decades, i.e., $46 \%$ and $42 \%$, respectively; however, a low frequency of this type of deformity was observed among those in their 2 nd and 8 th decades, i.e., $21 \%$ and $19 \%$, respectively. BC was frequently observed (40\%) among those in their 4th decade; FO (22\%) was frequently observed 
Table 7. Disk Deformities for Bi-Lateral Symptoms Joints According to Sex and Age

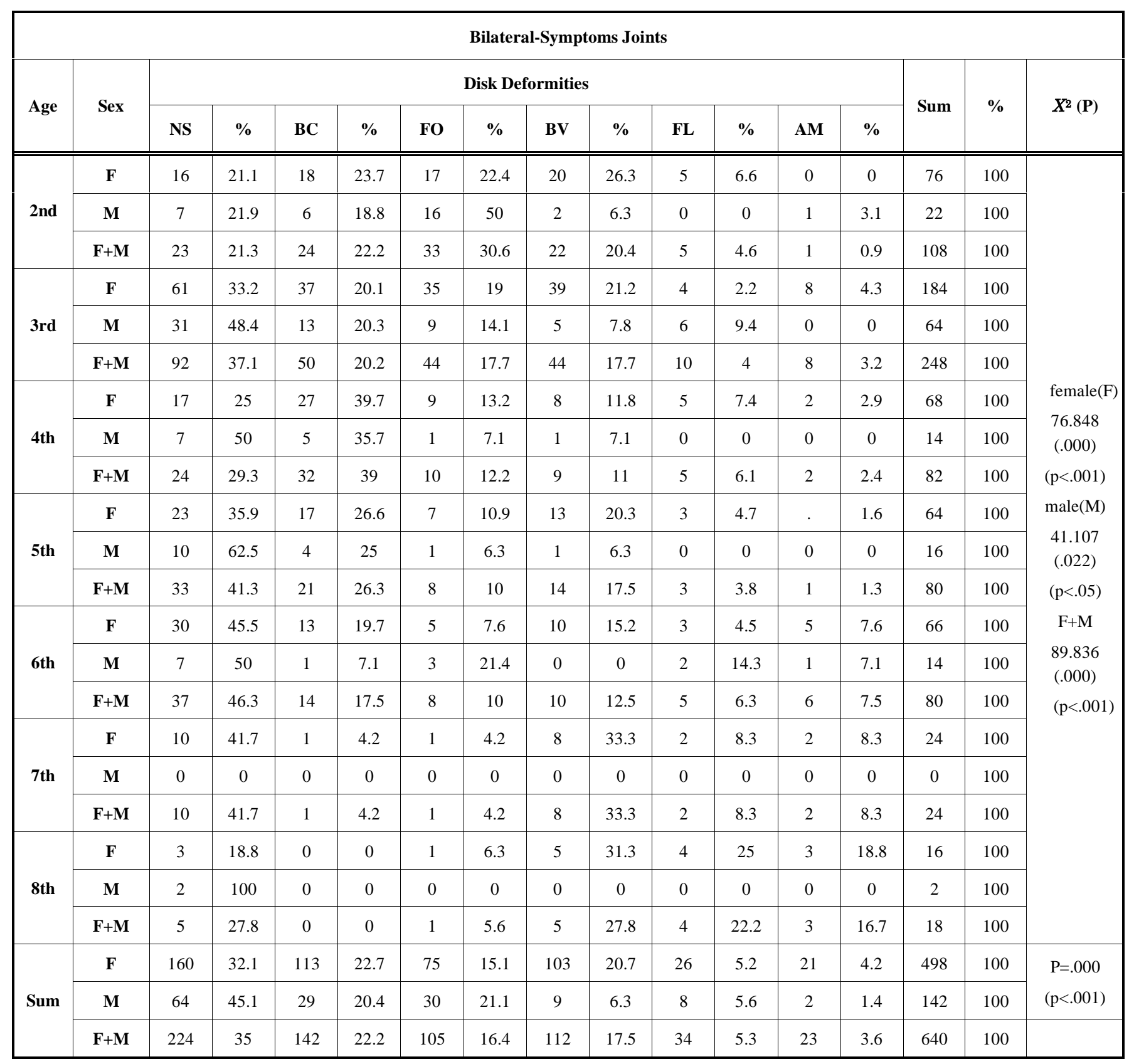

among those in their 2nd decade; and BV (33\%) was frequently observed among those in their 7 th decade. FL $(25 \%)$ and AM (19\%) showed high frequencies among subjects in their 8 th decade. There were statistically significant differences in terms of the frequency of disk deformity according to age in males $(\mathrm{p}<0.001)$. In males, NS $(45 \%)$ exhibited the highest frequency, followed by FO (21\%) and BC (20\%). As regards the frequency of disk deformity according to age, NS was found to be frequent among those in their 5th decade $(63 \%)$ than among those in the other age groups, whereas those in their 2nd decade (22\%) showed the lowest frequency of disk deformity. FO was the most frequent among those in their teens $(50 \%)$, while $\mathrm{BC}$ was the most frequently observed among those in their thirties $(36 \%)$. There were statistically significant differences among males in terms of the frequency of disk deformity according to age $(\mathrm{p}<0.05)$. As regards the assessment of all patients together, NS (3\%) was the most frequent observed, followed by BC (22\%), BV $(17 \%)$, and FO (16\%). As regards the assessment of disk deformity with respect to age, NS was more frequently observed among those in their 7 th decade $(46 \%)$ than it was among the other age groups, whereas it was infrequently observed among those in their teens $(21 \%)$. BC was more frequent among those in their 4th decade (39\%), whereas BV was more frequent among those in their 7 th decade $(33 \%)$ than it was in the other age groups. Subjects in their 2nd decade of life exhibited a remarkably higher frequency of FO $(31 \%)$ than did those in the other age groups. There were statistically significant differences in terms of the frequency of disk deformity according to age in the group that included 
all patients $(\mathrm{p}<0.001)$. As regards the distribution of disk deformities among males and females, significant differences were observed, i.e., $45 \%$ and $32 \%$ in the NS group, $21 \%$ and $15 \%$ in the FO group, and $6 \%$ and $21 \%$ in the BV group, respectively $(\mathrm{p}<0.001)$.

Frequency of Disk Deformity in Three Experimental Groups: Asymptomatic, and Unilaterally and Bilaterally Symptomatic Joint Groups (Two-Way ANOVA)

As regards disk deformity among asymptomatic subjects, and those with unilateral, and bilateral symptoms among males, females, and all patients considered together, there were significant interactive effects with respect to disk position by group $(\mathrm{p}<0.01$ for males; $\mathrm{p}<0.05$ for females; and $\mathrm{p}<0.001$ for the group of all patients). These results demonstrated significant differences in terms of the frequency of disk positions among asymptomatic subjects, as well as among the unilaterally and bilaterally symptomatic groups.

\section{Disk Deformities According to Disk Position}

In the asymptomatic joint group, males and females showed frequencies of NS-NS of $69 \%$ and $57 \%$, respectively; thus, the frequency of NS-NS among all patients was $60 \%$. Females showed a $9 \%$ frequency of CA-BV, while males showed a $6 \%$ frequency of PL-BC; therefore, the entire patient showed an $8 \%$ frequency of CA-BV. Joints with disk displacement, but without disk recapture, had frequency of $36 \%, 50 \%$, and $47 \%$, respectively, among males, females, and all patients. Among the joints showing disk displacement, those exhibiting complete disk displacement $(\mathrm{CM}+$ $\mathrm{CL}+\mathrm{CA})$ showed respective frequencies of $58 \%, 72 \%$, and $69 \%$ (Table 8, Fig. 3).

Table 8. Disk Deformities According to Disk Positions in the No Symptom Joints Group

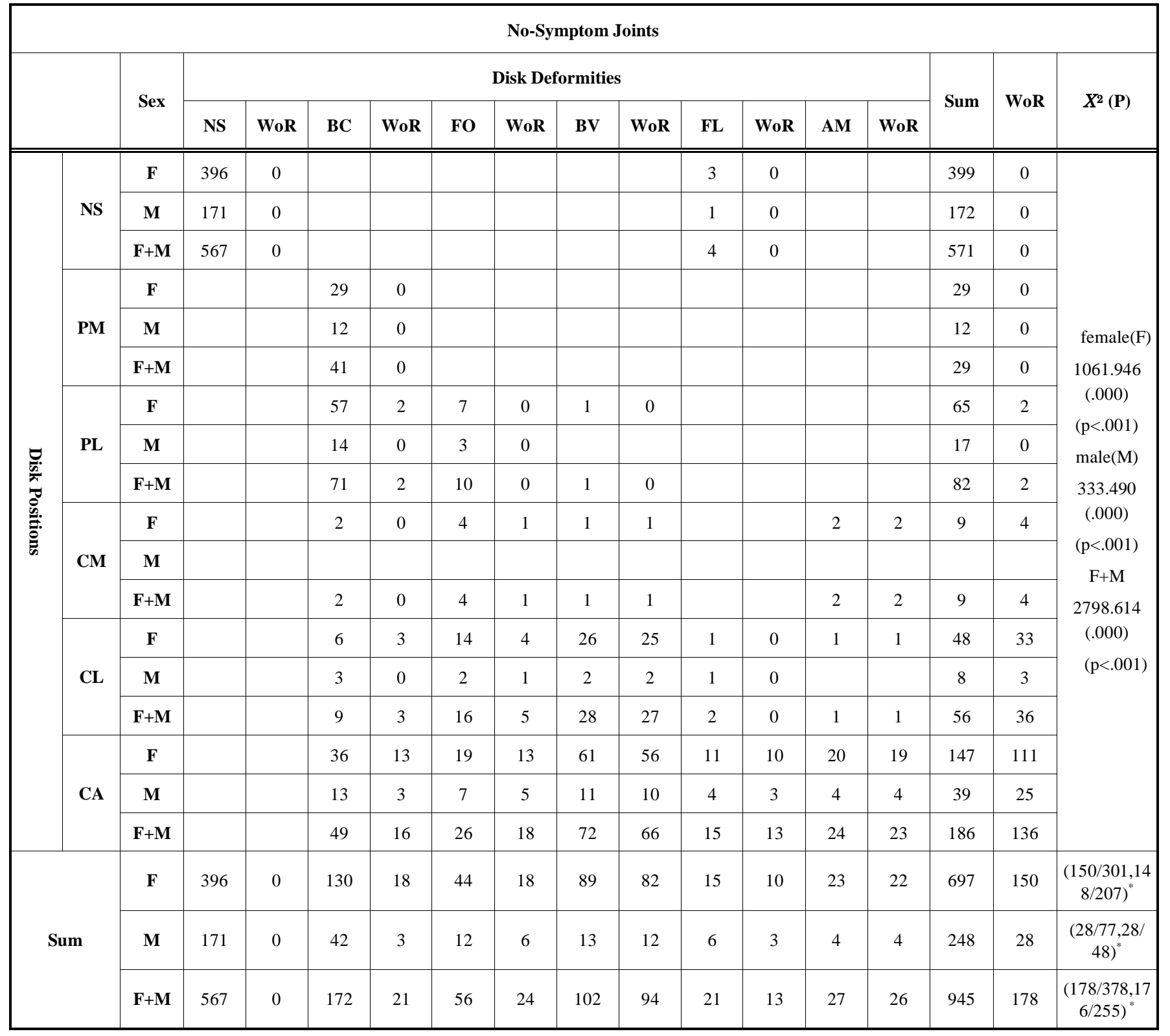

$(\mathrm{A} / \mathrm{B}, \mathrm{C} / \mathrm{D})^{*} ; \mathrm{A}=$ the numbers of $\mathrm{WoR}, \mathrm{B}=$ the number of showing disk displacement $\mathrm{C}=$ the numbers of $\mathrm{WoR}, \mathrm{D}=$ the number of complete disk displacement $(\mathrm{CM}+\mathrm{CL}+\mathrm{CA})$. 


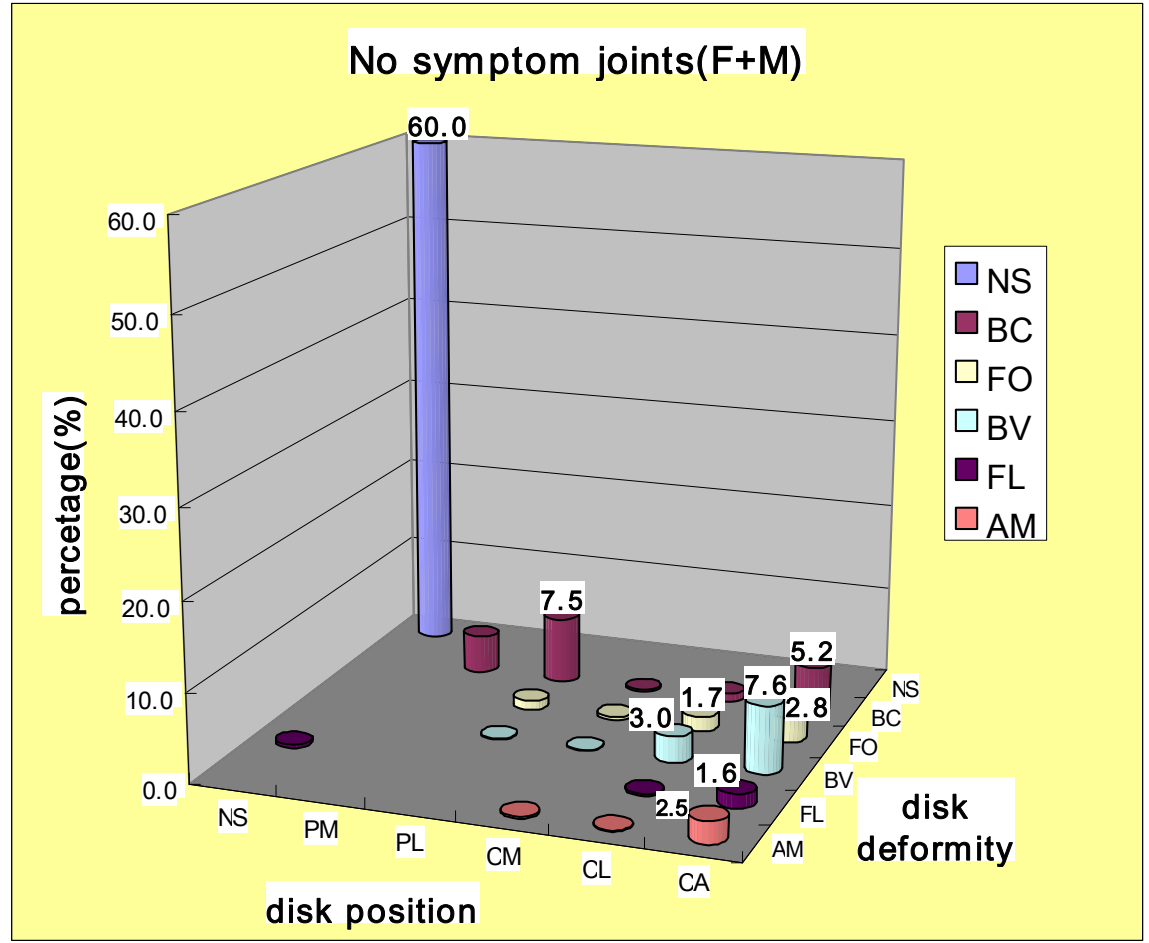

Fig. (3). Disk deformities according to disk positions in the no symptom joints group.

In the unilaterally symptomatic joint group, males and females showed frequencies of NS-NS of $48 \%$ and $21 \%$, respectively; thus, the total frequency was $28 \%$ among all patients. Females showed $15 \%$ frequency of $\mathrm{CA}-\mathrm{BV}$ and a $10 \%$ frequency of both CL-FO and CA-FO. Males showed a $10 \%$ frequency of CL-FO and an $8 \%$ frequency of PL-BC. The group of all patients showed a $13 \%$ frequency of $\mathrm{CA}-$ $\mathrm{BV}$ and a $10 \%$ frequency of both CL-FO and CA-FO. Joints with disk displacement but without disk recapture had frequencies of $49 \%, 67 \%$, and $64 \%$ among male, female, and all patients, respectively. Among subjects with this latter type of joint, those with complete disk displacement $(\mathrm{CM}+\mathrm{CL}+\mathrm{CA})$ showed respective frequencies of $59 \%, 75 \%$, and $72 \%$ (Table 9, Fig. 4).

In the bilaterally symptomatic joint group, the respective male and female frequencies of NS-NS were $44 \%$ and $33 \%$, thus, the total frequency was $36 \%$ all of patients. A $12 \%$ frequency of CA-BV, and a $10 \%$ frequency of both CL-FO and PL-BC were observed among females. Males showed an $11 \%$ frequency of CA-FO and a $10 \%$ frequency of CL-FO. The group including all patients showed a $10 \%$ frequency of $\mathrm{CA}-\mathrm{BV}$, an $8 \%$ frequency of CL-FO, and an $8 \%$ frequency of PL-BC. Joints exhibiting disk displacement, but which did not show disk recapture, had frequencies of $30 \%, 56 \%$, and $51 \%$ among males, females, and all patients, respectively. Among the subjects whose joints exhibited this latter type of displacement, those with complete disk displacement $(\mathrm{CM}+\mathrm{CL}+\mathrm{CA})$ had frequencies of $39 \%, 69 \%$, and $64 \%$, respectively (Table 10, Fig. 5).

\section{DISCUSSION}

To arrive at an adequate diagnosis of and appropriate treatment for TMD, it is necessary that the morphological and functional evaluation of the anatomical structure of the
TMJ is accurate, as based on the examination of imaging studies, as well as patient's clinical manifestations. Conventional radiography, conventional tomography, computerized tomography (CT), athrography, and MRI are primarily used in image-based examinations of TMD. Conventional radiography enables the assessment of general degenerative or traumatic bone changes, as well as that of the range of motion of the mandibular condyle. However, with this mode of analysis, it remains difficult to determine the correct position of the mandibular condyle, due to seriously distorted phases and excessive overlap with other anatomical structures [22]. Both conventional tomography and CT are useful for observing abnormalities in bone tissue due to developmental deformities, trauma, and tumors, but they are only limited used for observing disk displacement [23-25]. Moreover, although athrography is known to be useful for determining disk position indirectly, it has a number of disadvantages, namely, it is an invasive procedure that employs contrast media and exposes patients to high doses of radiation [26]. MRI is recognized as being very effective for determining disk shape and position, as well as and bone changes. In addition, MR imaging is non-invasive and does not involve exposing the patient to radiation; with this technology, excellent softtissue contrast can be obtained, even without the use of contrast media [26, 27]. Therefore, we used MRI, in the present study, as it was regarded as the best diagnostic imaging approach for diagnosing TMD. Here, we aimed to identify correlations between features seen on MR images with morphological and functional abnormalities in the TMJ region of TMD patients.

In this context, it should be noted that it is very important to first define the normal superior position of the disk when using MRI to determine disk position during mouth closing. Orsini et al. [19] reported the results of a comparative study of four diagnostic criteria; they found that the use of an in- 
Table 9. Disk Deformities According to Disk Positions in the Uni-Lateral Symptoms Joints Group

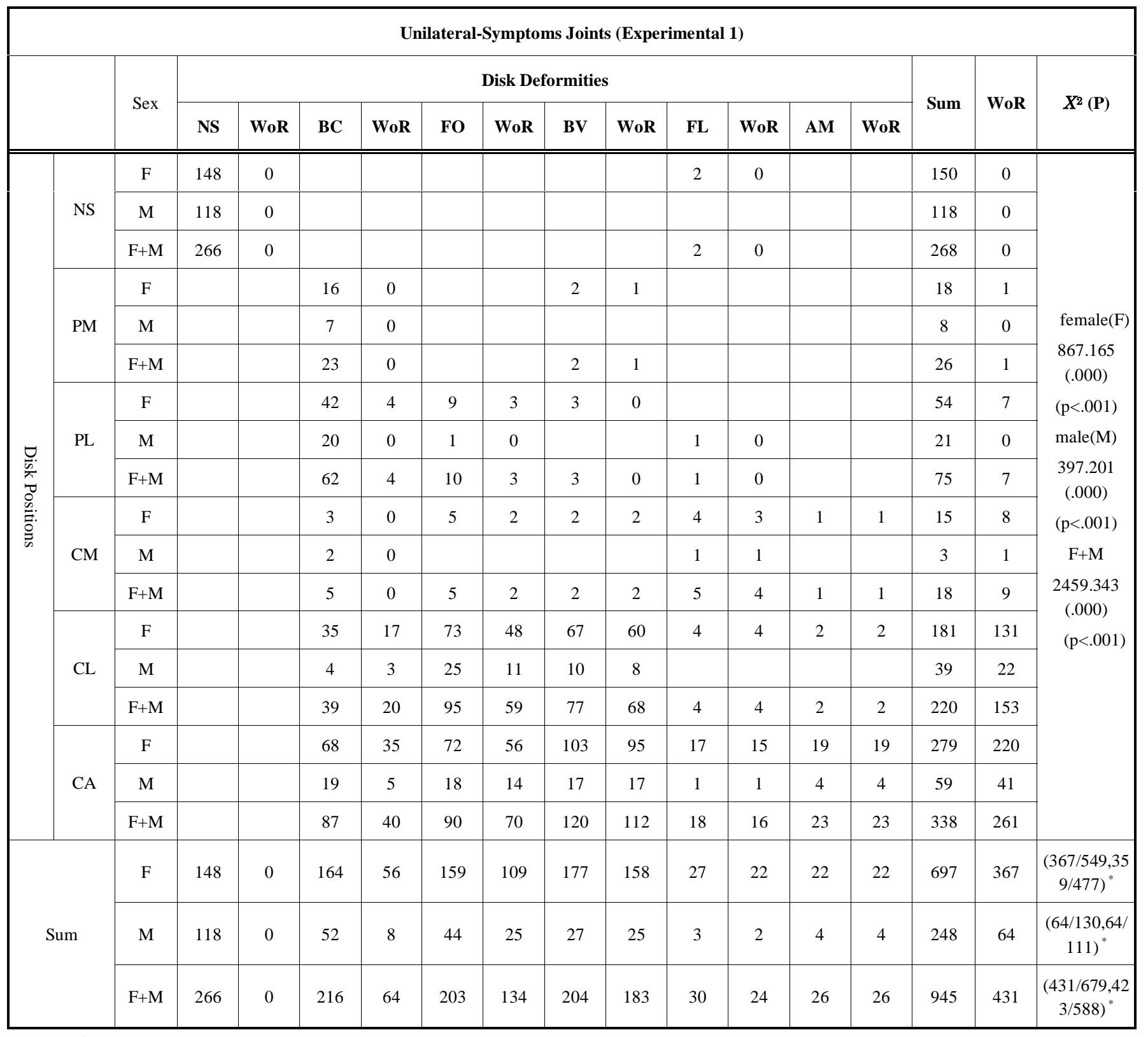

$(\mathrm{A} / \mathrm{B}, \mathrm{C} / \mathrm{D})^{*} ; \mathrm{A}=$ the numbers of WoR, $\mathrm{B}=$ the number of showing disk displacement $\mathrm{C}=$ the numbers of WoR, $\mathrm{D}=$ the number of complete disk displacement $(\mathrm{CM}+\mathrm{CL}+\mathrm{CA})$.

termediate criterion can reduce the false-positive rate. Tasaki et al. [16] suggested a nine-item system of classification based on disk displacement. Larheim et al. [17] further subdivided this system of classification into an eleven-item classification system that included complete disk displacement and partial disk displacement. We used the intermediate zone criterion as a standard for the normal superior position of the disk, and we determined disk positions in order to distinguish between partial and complete disk displacements; moreover, in the assessment of disk displacement, we differentiated between the mesial and lateral structures of the condyle in order to obtain more detailed information about disk displacement. However, we did not observe any coronal images, and therefore we were unable to avoid the possibility of obtaining false-negative results, which was determined as a finding of a normal superior position for a true lateral dis- placement [28, 29]. In the present study, instead of using only a single landmark as an anatomical index in order to determine disk position, varied indexes were used according to the images. Drace et al. [18] insisted that a clear boundary was observed between the disk and its posterior tissue in $60 \%$ of TMJs studied during mouth closing; likewise, $70 \%$ of the cases evaluated showed such a boundary during partial mouth opening, and $94 \%$ did so in association with the maximum mouth opening. Moreover, as it is possible for disk displacement to result in the alteration of the posterior tissue, a division can be obscured with changes in the signal intensity on MRI $[30,31]$. The boundary between the disk and the retrodiskal tissue can become obscured, particularly in cases involving the closed mouth, upon MRI used in clinical practice. Therefore, extended criteria for the determination of disk position must be taken into account, rather than 


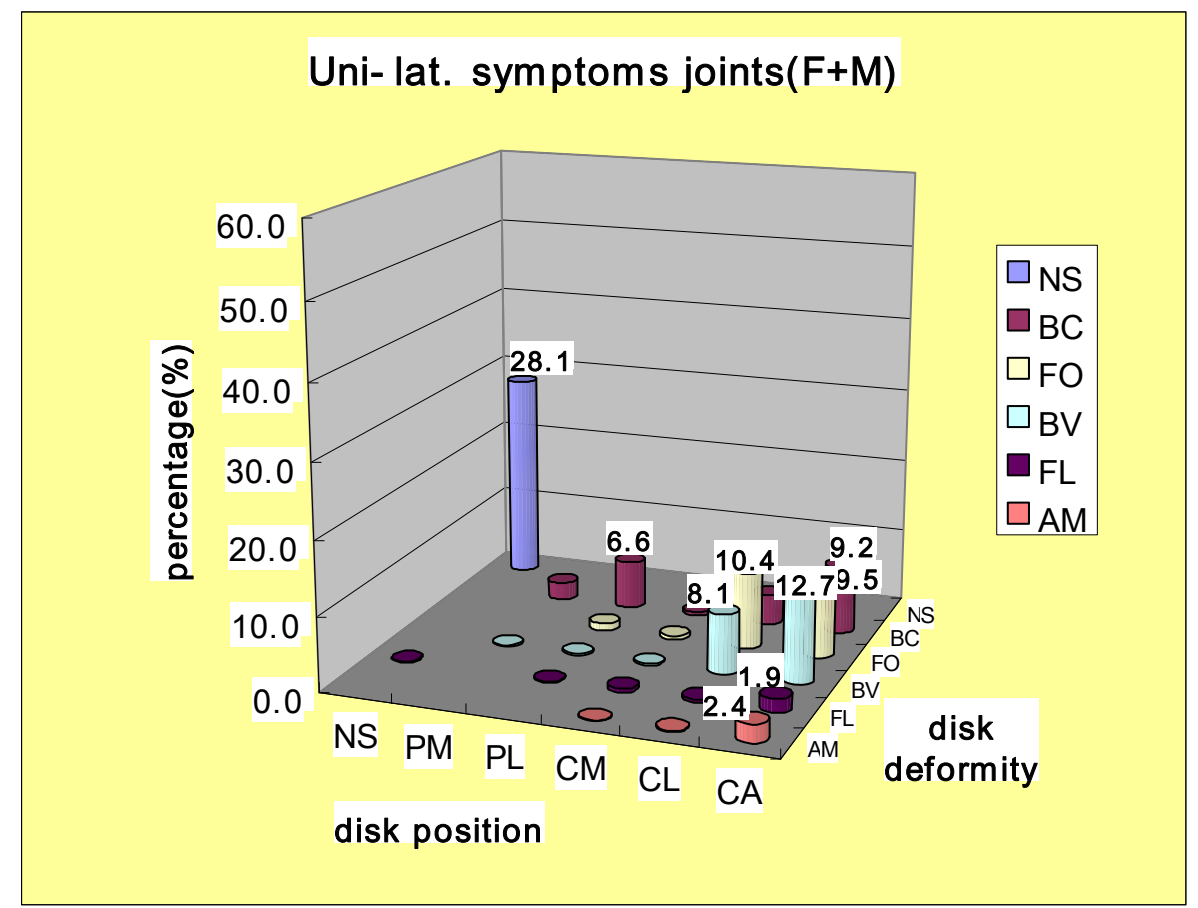

Fig. (4). Disk deformities according to disk positions in the uni-lateral symptoms joints group.

limiting evaluation to boundary of the disk, when determining the extent of disk displacement.

As regards the angular measurement of disk position, Rammelsbeg et al. [20], suggested that over 15 degrees be included in assessing the difference between the inside and the outside of disk position; in addition, over 70 degrees should be included in the category of CA, despite any difference between inside and outside of disk position. In identifying disk deformities in the TMJ, Heffez and Jordan [32] categorized disk configurations as shapes (e.g., bow-tie, straight, funnel, bulge, and $\mathrm{Y}$ shapes) using cephalometric arthrograms and histopathologic sagittal sections. Using MRI, Murakami et al. [33] employed the categories of biconcave, biplanar, hemiconvex, biconvex, and folded. However, there appeared to be a general lack of functional assessments among the previous reports, which focused merely on the morphology of disk using MR imaging. The present system of categorization emphasized in particular the length of the displaced disk in order to evaluate disk configurations; we also concentrated on abnormalities in the intermediate zone, which is an important anatomical structure in this context, and on alterations of the anterior and posterior bands. Although when determining the type of disk configuration, we observed differences between the mesial and distal areas according to disk position and the direction of observation, we were unable to fully account for these variations. No statistically significant differences were observed in terms of the distribution of disk positions among asymptomatic joints according to age, regardless of gender. NS showed a frequency of $60 \%$, and that of CA was $20 \%$, whereas CL was remarkably less frequency in the asymptomatic joint group than that in the unilaterally and bilaterally symptomatic joint groups. As regards comparisons between males and females, statistically significant differences were observed, i.e., NS was not frequently observed among females, whereas high frequencies of both CL and CA were observed among fe- males. Upon comparison of the frequency of disk deformities among females by age, it was found that those in their 4 th decade showed the lowest frequency of NS (44\%), and the frequency of NS was likely to be high in subjects younger or older than those in their thirties. In contrast, subjects in their 4th decade showed the highest frequency of $\mathrm{BV}$, whereas the frequency of $\mathrm{BV}$ was likely to be low among subjects younger or older ones than those in their thirties. Subjects ranging from their teens to their thirties showed higher frequencies of $\mathrm{BC}$ and $\mathrm{FO}$ than did those in the other age groups. There were statistically significant differences observed among females, but not among males in this regards. Statistically significant differences were observed between males and females, i.e., less frequent NS and more frequent $\mathrm{BV}$ were observed among females. Among those subjects with complete disk displacement, disk displacement without reduction accounted for $69 \%$ of the cases. Thus, disk displacement without disk reduction was found to occur frequently.

As regards disk positions in the unilaterally symptomatic joint group, females showed the highest frequency of CA (40\%), followed by CL (26\%) and NS (21\%), whereas males showed the highest frequency of NS $(48 \%)$, followed by CA (24\%) and CL (16\%). With respect to age, females in their teens showed a higher frequency of CL (40\%), but a lower frequency of NS (10\%) than did subjects in the other age groups. In addition, males in their teens decade showed higher frequencies of both CL and CA, i.e., $36 \%$ and $31 \%$, respectively, and a lower frequency of NS (22\%) than did the other age groups. NS was more frequently observed among females in the 7th (30\%) and 3rd (26\%) decade of life, and more frequently among males in the 7 th (67\%) and 8 th $(76 \%)$ decades of life, than in the other age groups. Thus, males showed a higher frequency of NS in the older age groups than did females. Statistically significant differences were observed regardless of gender, and also between males 
Table 10. Disk Deformities According to Disk Positions in the Bi-Lateral Symptoms Joints Group

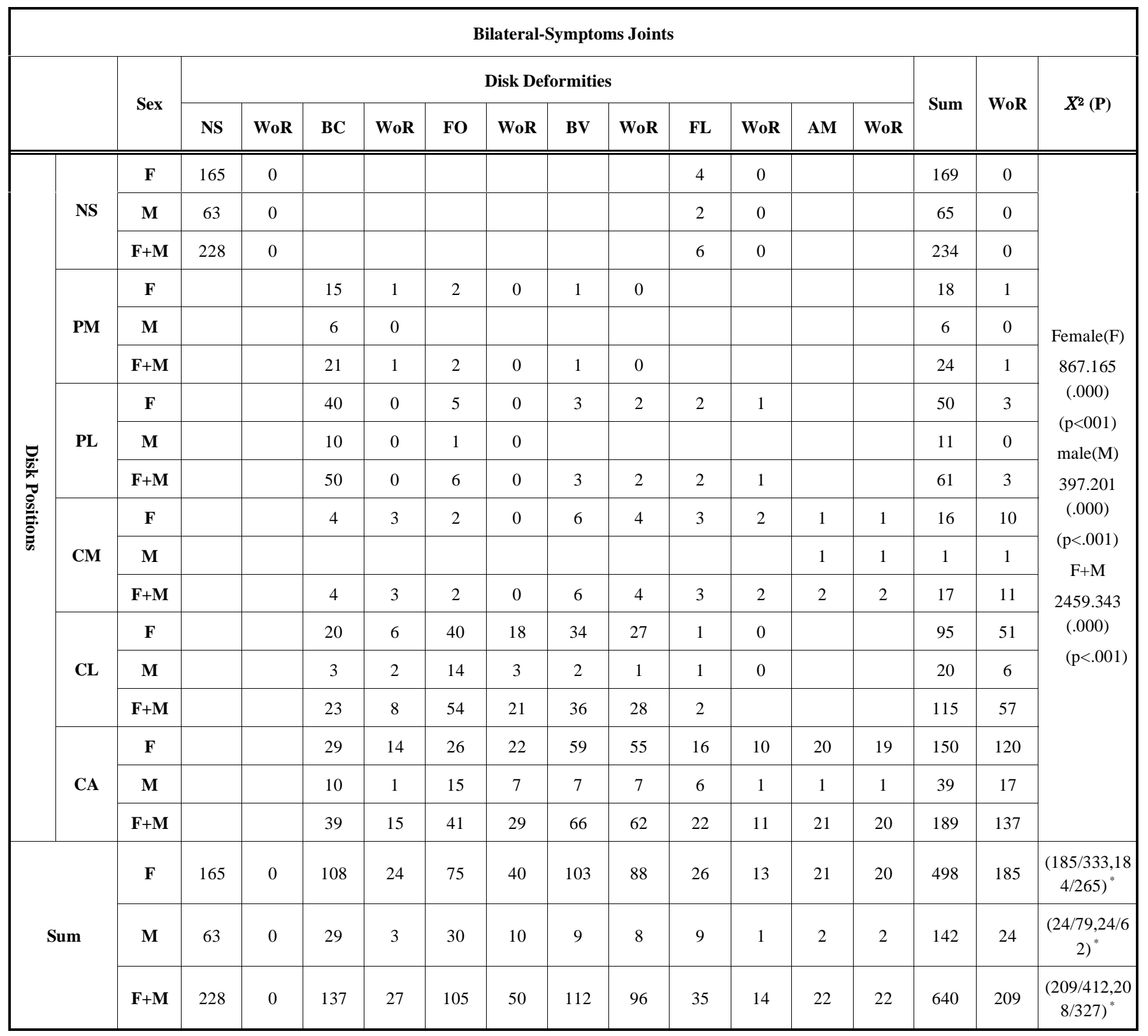

$(\mathrm{A} / \mathrm{B}, \mathrm{C} / \mathrm{D})^{*} ; \mathrm{A}=$ the numbers of $\mathrm{WoR}, \mathrm{B}=$ the number of showing disk displacement $\mathrm{C}=$ the numbers of $\mathrm{WoR}, \mathrm{D}=$ the number of complete disk displacement $(\mathrm{CM}+\mathrm{CL}+\mathrm{CA})$.

and females with respect to disk positions among those in the unilaterally symptomatic joint groups.

As regards the distribution of disk deformities, females showed the highest frequency of BV (25\%), followed by BC $(24 \%)$, FO (23\%), and NS (21\%); males showed the highest frequency of NS $(48 \%)$, followed by $\mathrm{BC}(21 \%)$ and $\mathrm{FO}$ $(18 \%)$. The assessment of these frequencies in terms of age revealed that females in their teens more frequently exhibited BC $(34 \%)$ and FO (36\%), and less frequently exhibited NS $(10 \%)$ and BV $(16 \%)$, than did those in the other age groups; however, males in their teens showed a high frequency of FO $(46 \%)$, but a low frequency of NS (22\%). A high frequency of BV was observed among females in their 6th decade $(38 \%)$ and among males in their 4th decade $(25 \%)$. Males showed a higher frequency of NS than did females, a higher frequency of NS was observed among males in their 5 th $(77 \%)$ and 8 th decades $(74 \%)$ than was observed among in the other age groups. The incidence of AM tended to be proportional to aging in females. There were statistically significant differences in terms of disk deformities regardless of gender, but significant differences were also observed between males and females. Among those subjects with complete disk displacement, disk displacement without reduction accounted for $72 \%$ of the cases. Thus, disk displacement without disk reduction was frequently observed.

As regards disk position in the bilaterally symptomatic joint group, females showed the highest frequency of NS (34\%), followed by CA (30\%) and CL (19\%), and males showed the highest frequency of NS (46\%), followed by CA (28\%) and CL (14\%); these results were thus not indicative 


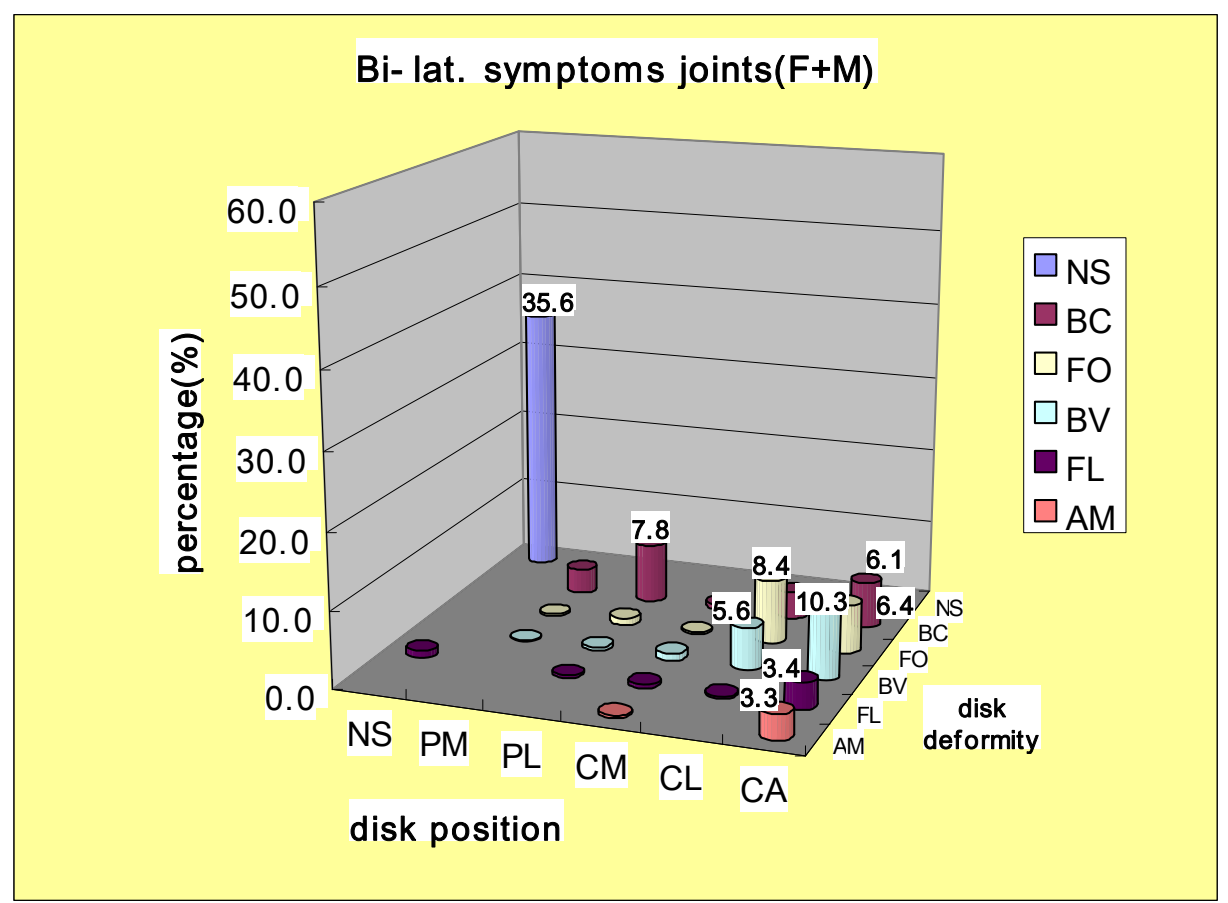

Fig. (5). Disk deformities according to disk positions in the bi-lateral symptoms joints group.

of a significant difference between males and females. As regards these frequencies with respect to various age groups, females in their teens showed higher frequencies of CA (34\%) and CL (28\%), but a lower frequency of NS (21\%), than did those in the other age groups, whereas males in their teens showed a higher frequency of CA (53\%), but a lower frequency of NS (22\%) than did those in the other age groups. CA was frequent among females in the 7 th $(42 \%)$ and 8th $(56 \%)$ decades of life, and NS was very frequent among older subjects, regardless of gender. There were statistically significant differences in terms of frequency of the distribution of various disk positions according to age group, regardless of gender.

As regards disk deformity, NS (32\%) was the most frequent among females, followed by BC (23\%), BV (21\%), and FO (15\%), whereas NS (45\%) was the most frequent among males, followed by FO (21\%), BC (20\%), and BV $(6 \%)$. As regards frequency according to age group, NS was found to be frequent among females in the 6th (46\%) and 7th (42\%) decades of life, but it was not frequent among those in their 2nd (21\%) and 8th (19\%) decades of life; however, NS was highly frequent among males in the 5th $(63 \%)$ decade, but had the lowest frequency among those in their 2nd (22\%) decade of life. BC was frequently observed among both males and females in their thirties, i.e., $36 \%$ and $40 \%$, respectively. FO was very frequent among both males and females in their teens, i.e., $50 \%$ and $22 \%$, respectively. There were significant differences in terms of disk deformities regardless of gender, but significant differences were also observed between males and females. Among those with complete disk displacement, disk displacements without reduction accounted for $64 \%$ of the cases. Thus, disk displacement without disk reduction was frequently observed.

In terms of the partial disk displacement (PM and PL) of disk positioning, no significant difference was observed in terms of frequency in the asymptomatic joint group (4\% and
$9 \%$ ), unilaterally symptomatic joint group (3\% and $8 \%$ ), or bilaterally symptomatic joint group (4\% and $10 \%)$, which appeared to indicate that partial disk displacement is on the borderline in terms of its association with clinical symptoms. $\mathrm{CM}$ had less than a 3\% frequency of expression in all three experimental groups, and was therefore quite different from CL $(6 \%, 23 \%$, and $18 \%$ in the asymptomatic group and in the unilaterally and bilaterally symptomatic joint groups, respectively). Although CA was most frequently expressed with disk displacement in all three experimental groups, the most remarkable increase was observed in the frequency of $\mathrm{CL}$, compared with the asymptomatic joint group. This finding demonstrates that $\mathrm{CL}$ is closely related to clinical symptoms, which had a high frequency of expression among those in their 2nd decades of life. A higher frequency of positive findings was obtained with MRI among those in their 2nd decades of life than among those in the other age groups; this result may provide support for the report by Isberg et al. [4], namely, that the onset of disk displacement frequently takes place during the teenage years.

The frequencies of PL and CL were higher than those of $\mathrm{PM}$ and CM. This finding strongly suggests that disk displacement tends to occur laterally originate in a lateral aspect of joint. In this respect, Wilkinson [34] reported observing differences in the attachments inside and outside of the disk and external pterygoid muscle; furthermore, Schmolke [35] reported finding an association between the masseter muscle and the joint capsule. Ben Amor et al. [36] reported that there were two different anatomical types of lateral disk attachment. One of these two types, i.e., that with an indirect lateral junction attached behind and below the pole through a sheet of fibrous tissue, had a significantly lower breaking strength point than did the other type, i.e., that with a direct lateral junction. Kino et al. [37] reported that most collagen fibers of the posterior band of the disk were attached to the condylar pole, and no diskal attachment was observed in the 
posterior aspect of the condyle. Another report noted an absence of collagen fiber to form a direct connection between the disk and the articular fossa. Scapino $[38,39]$ insisted that the posterior band and retrodiskal tissue became thinner in the lateral region of the TMJ, which was demonstrative of the parallel-fibered organization of the mesial region, instead of a form interwoven in a fan-like radiation.

The factors mentioned in this context may cause the lateral region of the TMJ, which is anatomically weaker than the mesial region, to be less resistant to external forces such as trauma; in addition, the lateral region may be sufficiently weak to facilitate disk displacement, may exhibit only slight restoration to a normal superior position, and may exhibit a high frequency of both PL and CL, as was demonstrated in present study.

$\mathrm{BC}$ showed the highest rate of expression in all three experimental groups in terms of the incidence of disk deformities; however, the unilaterally and bilaterally symptomatic joints tended to decrease somewhat with respect to $\mathrm{BC}$ and increase with respect to $\mathrm{BV}$; moreover, these groups showed the greater increases in FO than did the asymptomatic joint group. This finding indicates that $\mathrm{FO}$ is more closely related than is $\mathrm{BC}$ to the manifestation of clinical symptoms. Although the unilaterally and bilaterally symptomatic joint groups showed a high frequency of CA in terms of disk position, and a high frequency of $\mathrm{BC}$ in terms of disk deformity, they both showed a greater increase in the frequencies of CL and FO than did the asymptomatic joint group. There were no significant difference in terms of the existence of disk recapture between the unilaterally and bilaterally symptomatic joints and the asymptomatic joints, which most likely indicates that disk recapture fails to be advantageous in relation to clinical symptoms. Subjects in their teens showed the highest frequency of CL with respect to disk position and FO with respect to disk deformity, both of which tended to decrease among older subjects, most likely indicating spatial and morphological changes toward $\mathrm{CA}$ and $\mathrm{BV}$ with the passage of time. In order to determine how long it would take for these changes to occur and how they might be clinically manifested, longitudinal study will be necessary. However, since the asymptomatic joint group with disk displacement and deformities showed a high frequency of $\mathrm{CA}-\mathrm{BV}$, it appears that these factors contribute to the spatial and morphological changes into $\mathrm{CA}$ and $\mathrm{BV}$ that are associated with a loss of clinical symptoms. Moreover, $69 \%$ of the joints with complete disk displacement (CM+ $\mathrm{CL}+\mathrm{CA}$ ) showed no disk recapture, which was also likely to be related to a loss of symptoms. Subjects in their teens showed a higher correlation between disk position and deformity upon MRI of TMD, regardless of gender, than did subjects in the other age groups; in addition, females showed a higher correlation in this regard than did males. Moreover, older males showed a lower correlation between disk position and deformity than did younger males. The unilateral and bilateral symptom joint groups showed a high frequency of CA with respect to disk position and BV with respect to disk deformities, but showed a greater increase in the frequency of CL and FO than did those in the asymptomatic joint group. Although in the present study we failed to reveal any remarkable differences in terms of disk positions and disk deformity, it is thought that changes in disk positions and deformities would be necessary in cases of TMJ with disk displacement in order to alleviate symptoms, considering the self-limitation of TMD symptoms over time. Maintenance is necessary without additional anterior disk displacement and disk deformation through strong and healthy diskcondyle complex in case of partial displacement. Hence, physiological adaptations most likely accompany consistent disk displacement, and disk deformities may subsequently occur. Such physiological adaptations are thought to be induced in order to avoid having the disk interfere with the movement of the condyle; sufficient movement anterior to condyle should be viewed in all imaging slices, and changes are thought to occur in a biconvex manner, with decreases in the length of the disk. Morphological changes to the condyle should be observed in a further study, as they were not observed in the present cases. Such morphological changes are thought to be functional at the posterior slope of the mandibular condyle, which loses its functional role without disk recapture, by shortening the structure without indicating any changes in cortication. If the above mentioned changes are not induced or fail to occur, there is a greater possibility of long-term illness without any alleviation of symptoms.

In the present study, we thought that disk position and disk deformity might be different because of the age and gender, and then that they might influence clinical symptoms. We conducted the present comparative observation of MRI manifestations according to gender and age in order to identify the correlation between clinical manifestations and MRI findings in a large sample of patients with TMD. In concluded, the present study revealed significant differences in symptoms and MRI manifestations among patients with TMD according to gender and age; moreover, females and younger subjects showed a high correlation between clinical symptoms and MRI manifestations. Additional significant differences were observed between symptomatic and asymptomatic patients with respect to disk position and deformities.

\section{REFERENCES}

[1] The American academy of orofacial pain. Temporomandibular disorders: guideline for classification, assessment and management. In: Mcneil C, Ed. $2^{\text {nd }}$ ed. Chicago: Quintessence Publishing Co 1993; pp. 11-25.

[2] Bell WE. Tempormandibular disorders, classification, diagnosis, management. $3^{\text {rd }}$ ed. Chicago: Year Book Medical Publishers 1990; pp. 101-13.

[3] Pullinger AG, Seligma DA. TMJ osteoarthrosis: a differentiation of diagnostic subgroups by symptom history and demographics. J Craniomandib Disord 1987; 1: 251-6.

[4] Isberg A, Hagglund M, Paesani D. The effect of age and gender on the onset of symptomatic temporomandibular joint disk displacement. Oral Surg Oral Med Oral Pathol Oral Radiol Endod 1998; 85: 252-7.

[5] Katzberg R, Tallents R, Hayakawa K, Miller T, Goske M, Wood B. Internal derangements of the temporomandiblar joint: findings in the pediatric age group. Radiology 1985; 154: 125-7.

[6] Howard JA. Temporomandibular joint disorders, facial pain and dental problems of performing artists. In: Sataloaff R, Brandfonbrener A, Lederman R, Eds. Textbook of performing arts medicine. New York: Raven Press 1990; pp. 111-169.

[7] Lundh H, Westesson PL. Clinical signs of temporomandibular joint internal derangement in adult. Oral Surg Oral Med Oral Pathol 1991; 72: 637-41.

[8] Schiffman E, Friction JR. Epidermiology of TMJ and craniofacial pain. In: Friction JR, Kroening RJ, Hathaway KM, Eds. TMJ and craniofacial pain: diagnosis and management. St. Louis: IEA Pub 1988; pp. 1-10. 
[9] Huber MA, Hall EH. A comparison of the sign of temporomandibular joint dysfunction and occlusal discrepancies in symptomfree population of men and women. Oral Surg Oral Med Oral Pathol 1990; 70: 180-3.

[10] Barclay P, Hollender LG, Maravilla KR, Truelove EL. Comparison of clinical and magnetic resonance imaging diagnosis in patient with disk displacement in the temporomandibular joint. Oral Surg Oral Med Oral Pathol Oral Radiol Endod 1999; 88: 37-43.

[11] Margulles-Bonnet RE, Carpentier P, Yung JP, Defrennes D, Pharaboz C. Clincal diagnosis compare with findings of magnetic resonance imaging in 242 patients with internal derangement of the TMJ. J Orofac Pain 1995; 9: 244-53.

[12] Roberts CA, Katzberg RW, Tallents RH, Espeland MA, Handelman SL. The clinical predictability of internal derangements of the temporomandibular joint. Oral Surg Oral Med Oral Pathol 1991; 71: 412-4.

[13] Paesani D, Westesson PL, Hatala MP, Tallents RH, Brooks SL. Accuracy of clinical diagnosis for TMJ internal derangement and arthrosis. Oral Surg Oral Med Oral Pathol 1992; 73: 360-3.

[14] Emshoff R, Innerhofer K, Rudisch A, Bertram S. Relationship between tempormandibular joint pain and magnetic resonance imaging findings of internal derangement. Int J Oral Maxillofac Surg 2001; 30: 118-22.

[15] Haley DP, Schiffman EL, Lindgren BR, Anderson Q, Andreasen K. The relationship between clinical and MRI findings in patients with unilateral temporomandibular joint pain. J Am Dent Assoc 2001; 132: 476-81.

[16] Tasaki MM, Westesson PL, Isberg AM, Ren YF, Tallents RH. Classification and prevalence of temporomandibular joint disk displacement in patients and symptom-free volunteers. Am J Orthod Dentofacial Orthop 1996; 109: 249-62.

[17] Larheim TA, Westesson PL, Sano T. Temporomandibular joint disk displacement: comparison in asymptomatic volunteers and patients. Radiology 2001; 218: 428-32.

[18] Drace JE, Enzmann DR. Defining the normal temporomandibular joint: closed-, partially open-, and open-mouth MR imaging of asymptomatic subjects. Radiology 1990; 177: 67-71.

[19] Orsini MG, Kuboki T, Terada S, Matsuka Y, Yamashita A, Clark GT. Diagnostic value of 4 criteria to interpret temporomandibular joint normal disk position on magnetic resonance images. Oral Surg Oral Med Oral Pathol Oral Radiol Endod 1998; 86: 489-97.

[20] Rammelsberg P, Pospiech PR, Jager L, Pho Duc JM, Bohm AO, Gernet W. Variability of disk position in asymptomatic volunteers and patients with internal derangements of the TMJ. Oral Surg Oral Med Oral Pathol Oral Radiol Endod 1997; 83: 393-9.

[21] Choi YS, Hwang EH, Lee SR. A comparison of clinical symptoms and magnetic resonance images in temporomandibular joint disorders. Korean J Oral Maxillofac Radiol 2003; 33: 107-12.

[22] Aqulino SA, Matteson SR, Holland GA, Phillips C. Evaluation of condylar position from temporomandibular joint radiographs. J Prosthet Dent 1985; 53: 88-97.

[23] Paz ME, Katzberg RW, Tallents RH, Westesson PL, Proskin HM, Murphy WC. CT evaluation of the TMJ disc. Oral Surg Oral Med Oral Pathol 1988; 66: 519-24.
[24] Westesson PL, Katzberg RW, Tallents RH, Woodworth RE, Svensson SA. CT and MR of the TMJ: comparison with autopsy specimens. AJR Am J Roentgenol 1987; 148: 1165-71.

[25] Fava C, Gatti G, Cardes E, Parchetti R, Rocca G, Preti G. Possibilities and limits in identifying the TMJ articular meniscus with the CT scanner: a comparative anatomoradiological study. J Craniomandib Disord 1988; 2: 141-7.

[26] Westesson PL, Bronstein SL, Liedberg JL. Internal derangement of the temporomandibular joint: morphologic description with correlation to joint function. Oral Surg Oral Med Oral Pathol 1985; 59: 323-31.

[27] Drace JE, Young SW, Enzmann DR. TMJ meniscus and bilaminar zone: MR imaging of the substructure-diagnostic landmarks and pitfalls of interpretation. Radiology 1990; 177: 73-6.

[28] Katzberg RW, Westesson PL, Tallents RH, et al. Temporomandibular joint: MR assessment of rotational and sideways disk displacements. Radiology 1988; 169: 741-8.

[29] Tasaki MM, Westesson PL. Temporomandibular joint: diagnostic accuracy with sagittal and coronal MR imaging. Radiology 1993; 186: 723-9.

[30] Sano T. Recent developments in understanding temporomandibular joint disorders. Part 2: Changes in the retrodiscal tissue. Dentomaxillofac Radiol 2000; 29: 260-3.

[31] Laurell KA, Tootle R, Cunningham R, Beltran J, Simon D. Magnetic resonance imaging of the temporomandibular joint. Part II: comparison with laminographic, autopsy, and histologic findings. J Prosthet Dent 1987; 58: 211-8.

[32] Heffez L, Jordan S. A classification of temporomandibular joint disk morphology. Oral Surg Oral Med Oral Pathol 1989; 67: 11-9.

[33] Murakami S, Takahashi A, Nishiyama H, Fujishita M, Fuchihata H. Magnetic resonance evaluation of the temporomandibular joint disc position and configuration. Dentomaxillofac Radiol 1993; 22: 2057.

[34] Wilkinson TM. The relationship between the disk and the lateral pterygoid muscle in the human temporomandibular joint. J Prosthet Dent 1988; 60: 715-24.

[35] Schmolke C. The relationship between the temporomandibular joint capsule, articular disc and jaw muscles. J Anat 1994; 184 335-45.

[36] Ben Amor F, Carpentier P, Foucart JM, Meunier A. Anatomic and mechanical properties of the lateral disc attachment of the temporomandibular joint. J Oral Maxillofac Surg 1998; 56: 1164-7; discussion 1168-9.

[37] Kino K, Ohmura Y, Amagasa T. Reconsideration of the bilaminar zone in the retrodiskal area of the temporomandibular joint. Oral Surg Oral Med Oral Pathol 1993; 75: 410-21.

[38] Scapino RP. The posterior attachment: its structure, function, and appearance in TMJ imaging studies. Part 1. J Craniomandib Disord 1991; 5: 83-95.

[39] Scapino RP. The posterior attachment: its structure, function, and appearance in TMJ imaging studies. Part 2. J Craniomandib Disord 1991; 5: 155-66

(C) Choi et al.; Licensee Bentham Open.

This is an open access article licensed under the terms of the Creative Commons Attribution Non-Commercial License (http://creativecommons.org/licenses/ by-nc/3.0/) which permits unrestricted, non-commercial use, distribution and reproduction in any medium, provided the work is properly cited. 